\title{
Interactive endothelial phenotype maintenance and osteogenic differentiation of adipose tissue stromal vascular fraction SSEA-4+-derived cells
}

\author{
Silvia M. Mihaila ${ }^{1,2}$, Margarida F. Resende ${ }^{1,2}$, Rui L. Reis ${ }^{1,2}$, Manuela E. Gomes ${ }^{1,2}$ and \\ Alexandra P. Marques ${ }^{1,2 *}$ \\ ${ }^{1} 3 B$ Research Group, Biomaterials, Biodegradables and Biomimetics, University of Minho, Barco, GMR, Portugal \\ ${ }^{2}$ ICVS/3Bs, PT Government Associated Laboratory, Braga, Guimarães, Portugal
}

\begin{abstract}
Bone formation relies on complex processes that require well-orchestrated interactions between several cell types, such as bone-forming cells (osteoblasts, OBs) and endothelial cells (ECs). Their co-culture has been proved relevant to mimicking specific features of the bone niche. Here we propose the co-culture of microvascular-like ECs and pre-OBs, both derived from the SSEA- $4^{+}$cell subpopulation from the stromal vascular fraction of human adipose tissue (SSEA-4 ${ }^{+}$hASCs), to define the conditions in which cells synergistically communicate to support the full differentiation of pre-OBs and maintenance of the EC phenotype. Co-cultures of different ratios of the two cell types were established and maintained for up to 21 days in standard endothelial maintenance (ENDO) and osteogenic differentiation (OST) media, as well as in a mixture of these (MIX). The osteogenic maturation of pre-OBs (ALP activity, OPN and OCN expression, calcium deposition), the evolution of EC numbers (CD31 ${ }^{+}$cells) and maintenance of the endothelial phenotype (CD31 and $v W F$ expression, LDL uptake) were assessed throughout the culture time as a function of cell ratio and culture media. The results obtained demonstrate that EC number has a significant effect on the osteogenic differentiation of pre-OBs, depending on the medium used. While in ENDO medium the osteogenic differentiation was not observed, in the OST and MIX media it was attained at similar levels, except for the co-culture with a higher number of ECs in MIX medium. These findings demonstrate that the use of SSEA- $4^{+}$hASCs as a single-cell source is promising to attain 3D bone-like models with the potential to promote vascularized bone tissue regeneration. Copyright @ 2015 John Wiley \& Sons, Ltd.
\end{abstract}

Received 25 June 2015; Revised 29 July 2015; Accepted 15 September 2015

Keywords co-culture; SSEA-4-positive hASCs; endothelial cells; osteoblast-like cells; bone; vascularization

\section{Introduction}

Bone is a complex and highly specialized tissue that undergoes continuous regeneration and remodelling throughout life (Rodan, 1992). This intrinsic feature enables bone development and growth under normal physiological conditions, as well as in case of injury or trauma.

*Correspondence to: Alexandra P. Marques, Ave Park, Zona Industrial da Gandra, S. Cláudio do Barco 4806-909, Caldas das Taipas-Guimarães, Portugal. E-mail: apmarques@dep. uminho.pt
The prerequisite to maintaining this remarkable capability is an appropriate blood supply and the coupling of the vasculature with the bone remodelling cells. In fact, the intimate crosstalk between bone-forming (osteoblasts, OBs) and bone-resorbing (osteoclasts) cells and the microvascular endothelium orchestrates the spatial and temporal cellular activities that lead to bone regeneration (Grellier et al., 2009; Kaigler et al., 2006; Pirraco et al., 2010). Several in vitro studies have been investigating the mechanisms underlying this mutual interaction during bone formation, using various co-culture models of OBs and endothelial cells (ECs) as tools, to replicate the bone niche (Guillotin et al., 2008; Villars et al., 2002). 
Two dialogues were identified as essential for bone remodelling: a paracrine effect through growth factors, such as vascular endothelial growth factor (VEGF) (Street and Lenehan, 2009), insulin-like growth factors I and II (Huang et al., 2010) and bone morphogenetic proteins (BMPs) (Groeneveld and Burger, 2000), and a juxtacrine mechanism through gap-junctional communication (Villars et al., 2002).

Several research groups have been co-culturing osteogenic and vasculogenic cells to enable the occurrence of such dialogues, as well as the translation into complex $3 \mathrm{D}$ structures to recreate the native tissue. In the attempt to further mimic and eventually control the regenerative niche, the selection of cellular combinations has been significantly varied; ECs were co-cultured with mature OBs (Hofmann et al., 2008), precommitted osteoblast cells (pre-OBs) (Guillotin et al., 2008) or mesenchymal stem cells (MSCs) (Duffy et al., 2009; Koob et al., 2011; Ma et al., 2011; Rouwkema et al., 2009). Similarly, cell sources range from bone samples to obtain fully committed OBs, peripheral and umbilical cord blood to retrieve ECs or endothelial progenitor cells (EPCs), and bone marrow to isolate undifferentiated MSCs. However, in addition to some limitations associated with the availability/accessibility of cell sources, the ideal combination of cells and the respective conditions necessary to maximize their potential in a therapeutic approach are yet to be defined.

Adipose tissue has been widely exploited as a source of a myriad of cell types relevant in the context of tissue engineering (TE) and regenerative medicine. The relative easiness of harvesting adipose tissue (enabling low site morbidity and patient discomfort) and high cellular yield are among the factors that have increasingly favoured the use of adipose-derived stem cells (hASCs) over bone marrow MSCs. Previous studies (Astori et al., 2007; Rada et al., 2011) have also shown that the stromal vascular fraction (SVF) of the adipose tissue comprises several cellular subsets that, in comparison with the homogeneous population (hASCs), are characterized by an increased differentiation potential towards specific lineages. For instance, in the context of bone TE, STRO- $1^{+}$hASCs (Rada et al., 2011), CD105- hASCs (Levi et al., 2011), CD90 ${ }^{+}$ hASCs (Chung et al., 2013; Rada et al., 2011) and p75 hASCs (Rada et al., 2011) were shown to possess higher osteogenic differentiation potential than hASCs. Interestingly, few studies and with limited outcomes have reported the isolation of microvascular ECs from SVF (Hutley et al., 2001) or the differentiation of hASCs into the endothelial lineage (Fischer et al., 2009). In a previous study we showed that within the SVF of the adipose tissue resides a subpopulation that exhibits both osteogenic and endothelial differentiation capacity (Mihaila et al., 2013). This subpopulation, characterized by the expression of the pluripotency-associated marker SSEA-4 (SSEA- $4^{+}$hASCs), can be selected from SVF and gives rise to osteoblastic- and endothelial-like cells that can be further expanded into phenotypically stable populations. Interestingly, adipose tissue has never been explored from the perspective of establishing co-culture models relevant for bone regeneration. Considering the already mentioned gains of adipose tissue in the context of TE (Mihaila et al., 2013) and its exploitation for bone tissue regeneration (Chung et al., 2013; Levi et al., 2011; Mihaila et al., 2013; Rada et al., 2011), the use of SSEA-4 ${ }^{+}$hASCs as a single cell source of osteoblastic- and endothelial-like cells is of major relevance to further advancing the recreation of a microenvironment that potentiates bone regeneration. Under what conditions the potential of this subpopulation can be maximized is the question yet to be answered.

Previous studies have shown that the combination of less-differentiated osteoblastic cells (pre-OBs) with mature and fully differentiated ECs is the most effective in terms of osteogenic differentiation and endothelial tubelike formation features (Guillotin et al., 2008; Valenzuela et al., 2013). Moreover, a major concern regarding the preservation of the EC phenotype has been dictating the tested conditions (Bidarra et al., 2011). Thus, this study was designed under the hypothesis that SSEA- $4^{+}$hASCderived pre-OBs and SSEA- $4^{+}$hASC-derived ECs synergistically communicate to support the concomitant full differentiation of SSEA- $4^{+}$hASC-derived pre-OBs into an osteogenic phenotype and the maintenance of the SSEA$4^{+}$hASC-derived ECs phenotype. Co-cultures of different ratios of the two cell types were established and maintained for up to 21 days. Moreover, in order to confirm the cellular crosstalk and assess its overall effect over the osteogenic and endothelial phenotypes during the culture period, several culture media were tested and the involvement of VEGF evaluated.

\section{Materials and methods}

\subsection{Isolation of SVF}

Human abdominal subcutaneous adipose tissue samples were obtained from healthy females aged $42.3 \pm 15.9$ years undergoing lipoaspiration procedure. Samples were transported in phosphate-buffered saline (PBS; Sigma, Germany) supplemented with $10 \%$ v/v penicillinstreptomycin (Pen-Strep; $1000 \mathrm{U} / 1000 \mu \mathrm{g} / \mathrm{ml}$; Gibco, USA), under a protocol previously established between the Department of Plastic Surgery of Hospital da Prelada (Porto, Portugal) and the 3Bs Research Group (Guimarães, Portugal) and approved by the local Ethical Committee to assure that the requirements defined by the Helsinki Declaration guidelines regarding human rights, patients' informed consent and patients' anonymity were followed.

All tissue samples were processed within $24 \mathrm{~h}$ after the surgical procedure, as described elsewhere (Mihaila et al., 2013). Briefly, the lipoaspirates were digested with $0.05 \%$ w/v collagenase type IIA from Clostridium histolyticum (Sigma, Germany) in PBS for $45 \mathrm{~min}$ and under agitation in a shaking bath set at $37^{\circ} \mathrm{C}$. The digested tissue was filtered through a $200 \mu \mathrm{m}$ strainer, followed by 
centrifugation at $800 \times g$ for $10 \mathrm{~min}$ to remove the floating mature adipocytes and connective tissue. Lysis was performed with an in-house prepared lysis buffer, consisting of $155 \mathrm{mM} \mathrm{NH}_{4} \mathrm{Cl}$ (Merck, Germany), $5.7 \mathrm{mM}$ anhydrous $\mathrm{K}_{2} \mathrm{HPO}_{4}$ (Riedel-de-Haën, Germany) and 0.1 M EDTA (Sigma, Germany), in order to disrupt the red blood cells and further purify the crude cell aspirate. After several centrifugation and washing steps, the crude aspirate was resuspended in PBS and filtered through a $100 \mu \mathrm{m}$ cell strainer to ensure a single cell suspension consisting of the SVF of adipose tissue.

\subsection{SSEA-4 ${ }^{+}$hASCs subpopulation selection and culture}

\subsubsection{Immunomagnetic selection of SSEA-4 ${ }^{+}$ hASCS}

The SVF subpopulation expressing SSEA-4 (SSEA-4 ${ }^{+}$ hASCs) was isolated by immunomagnetic selection with SSEA-4 (clone MC813) antibody (Santa Cruz Biotechnology, USA)-coated Dynabeads ${ }^{\circledR}$ M-450 Epoxy beads (Invitrogen, USA), adapting the manufacturer's instructions as previously described elsewhere (Mihaila et al., 2013).

\subsubsection{Endothelial differentiation}

The freshly selected SSEA- $4^{+}$hASCs were filtered through a $100 \mu \mathrm{m}$ cell strainer to separate the single cells from the aggregates formed during coupling that might contain contaminating cells (fibroblasts, etc.). SSEA- $4^{+}$hASCs cells were immediately cultured in endothelial cell basal medium (EGM-2MV; Lonza, Switzerland) containing growth factors and 5\% fetal bovine serum (FBS). The cells were incubated in a humidified environment at $37^{\circ} \mathrm{C}$ with $5 \% \mathrm{CO}_{2}$, with culture medium replenishments every 3-4 days. For all experiments, SSEA-4 ${ }^{+}$hASC-derived ECs (microvascular-like endothelial cells) were used at passage 2 after $80 \%$ confluence and passaging with TrypLE ${ }^{\mathrm{TM}}$ Express (Invitrogen, USA).

\subsubsection{Osteogenic preconditioning}

Freshly selected SSEA-4 ${ }^{+}$hASCs were cultured in basal medium comprising minimum essential medium- $\alpha$ modification ( $\alpha$ MEM; Gibco, USA) supplemented with sodium bicarbonate $\left(\mathrm{NaHCO}_{3}\right.$; Sigma, Germany), $10 \%$ FBS (Gibco, USA) and 1\% Pen-Strep (100 U/100 $\mu \mathrm{g} / \mathrm{ml}$ ) for expansion. Osteogenic preconditioning was achieved by culturing SSEA-4 $4^{+}$hASCs at passage 1 for 7 days in osteogenic medium [basal medium supplemented with $10 \mathrm{~mm} \beta$-glycerophosphate ( $\beta \mathrm{GP}$; Sigma, Germany), $10^{-8} \mathrm{M}$ dexamethasone (Dex; Sigma, Germany), $50 \mathrm{mg} / \mathrm{ml}$ L-ascorbic acid 2-phosphate sesquimagnesium salt hydrate (AA; Sigma, Germany)] prior to initiation of the co-culture experiments.

\subsection{Co-culture set-up}

The SSEA- $4^{+}$hASC-derived ECs obtained from the SSEA$4^{+}$hASCs subpopulation were cultured together with osteogenic preconditioned SSEA- $4^{+}$hASCs $\left(\right.$SSEA-4 ${ }^{+}$ hASC-derived pre-OBs) at different cell ratios [SSEA-4 ${ }^{+}$ hASC-derived ECs:SSEA-4 ${ }^{+}$hASC-derived pre-OBs, 75\%:25\% (75:25), 50\%:50\% (50:50) and 25\%:75\% (25:75)] and in three different media [EGM-2 MV (ENDO), osteogenic (OST) and a combination of both (MIX)] as detailed in Table 1. Monocultures of $100 \%$ SSEA- $4^{+}$hASC-derived ECs (100:0) and 100\% SSEA-4 ${ }^{+}$ hASC-derived pre-OBs (0:100) were used as controls. All cells were used at passage 2 .

Cell suspensions at the defined ratios were seeded in 24-well plates at a density of $2 \times 10^{3}$ cells $/ \mathrm{cm}^{2}$ and in $500 \mu \mathrm{l}$ defined medium, which was changed twice weekly.

The morphology of the cells was evaluated during the culture at preselected time points (days 4, 7, 14 and 21), using a stereomicroscope Stemi 1000 (Zeiss, Germany).

In each experiment, each condition was always set up in triplicate. Each experiment was carried out three times independently, each time with SSEA- $4^{+}$hASCs derived from a different donor.

\subsection{Flow cytometry}

The phenotype of the cells used to set the co-cultures, after the endothelial differentiation (SSEA-4 ${ }^{+}$hASCderived ECs) and osteogenic pre-conditioning (SSEA-4 ${ }^{+}$ hASC-derived pre-OBs), was screened by flow cytometry, using the following antibodies: CD73-PE, CD34-PE and CD31-APC (all from BD Bioscience, USA) and CD105-FITC and CD90-APC (eBiosciences, USA). Cells were harvested with TrypLE ${ }^{\mathrm{TM}}$ Express (Invitrogen, USA) and $2 \times 10^{5}$

Table 1. Composition of the cell culture media used in the co-cultures

\begin{tabular}{|c|c|}
\hline Medium & Composition \\
\hline BASAL & $\alpha$ MEM supplemented with $\mathrm{NaHCO}_{3}, 10 \%$ FBS and $1 \%$ pen-strep $(100 \mathrm{U} / 100 \mu \mathrm{g} / \mathrm{ml})$ \\
\hline ENDO & $\begin{array}{l}\text { EGM-2 MV: each } 500 \mathrm{ml} \text { EBM-2 is supplemented with } 25 \mathrm{ml} \text { FBS, } 0.2 \mathrm{ml} \text { hydrocortisone, } 2 \mathrm{ml} \mathrm{hFGF-B,} 0.5 \mathrm{ml} \text { ascorbic acid, } \\
0.5 \mathrm{ml} \text { gentamicin-amphotericin, } 0.5 \mathrm{ml} \text { VEGF, } 0.5 \mathrm{ml} \text { Long R3-IGF-1, } 0.5 \mathrm{ml} \text { hEGF and } 0.5 \mathrm{ml} \text { heparin }\end{array}$ \\
\hline OST & Basal medium supplemented with $10 \mathrm{mM} \beta \mathrm{GP}, 10^{-8} \mathrm{M}$ Dex and $50 \mu \mathrm{g} / \mathrm{ml} \mathrm{AA}$ \\
\hline MIX & EGM-2 MV supplemented with $10 \mathrm{mM} \beta \mathrm{GP}, 10^{-8} \mathrm{M}$ Dex and $50 \mu \mathrm{g} / \mathrm{ml}$ AA \\
\hline
\end{tabular}

EBM-2, endothelial basal medium; hEGF, human epidermal growth factor; hFGF-B, human fibroblast growth factor - basic with heparin; hR3-IGF-1, human recombinant insulin-like growth factor; VEGF, vascular endothelial growth factor. 
cells in $2 \% \mathrm{w} / \mathrm{v}$ BSA/PBS in $0.1 \% \mathrm{w} / \mathrm{v}$ sodium azide were incubated with the antibodies, at the concentrations recommended by the manufacturer, for $30 \mathrm{~min}$ at room temperature in the dark. After incubation, the cells were washed with $2 \% \mathrm{w} / \mathrm{v}$ BSA/PBS and resuspended in acquisition buffer ( $1 \% \mathrm{v} / \mathrm{v}$ formaldehyde, $0.1 \% \mathrm{w} / \mathrm{v}$ sodium azide in PBS). At preselected time points, samples from all experimental groups were retrieved and incubated with CD31-APC, as described above, to quantify the percentage of SSEA- $4^{+}$hASC-derived ECs present in the culture.

Samples were analysed using a BD FACScalibur flow cytometer (BD Biosciences, USA). A minimum of $2 \times 10^{4}$ gated events was acquired and the cells of interest were gated in a forward vs side-scatter dot-plot with a linear scale, using FlowJo v. 10.0.6 software (Tree Star, USA). Histogram plots were used to determine the percentages of $\mathrm{CD}^{+} 1^{+}$(SSEA-4 $^{+}$hASC-derived ECs) and CD31 (SSEA- $4^{+}$hASC-derived pre-OBs).

\subsection{Endothelial phenotype analysis}

In addition to flow cytometry, the phenotype of SSEA-4 ${ }^{+}$ hASC-derived ECs was further analysed based on their capacity to form tubular-like structures on Matrigel and to uptake the acetylated low-density lipoprotein (ac-LDL) complex. This distinctive capacity of ECs was also used to label the SSEA-4 ${ }^{+}$hASC-derived ECs at preselected time points (days 4, 7, 14 and 21) of the co-culture, to obtain information about the cell distribution amongst the SSEA-4 ${ }^{+}$hASC-derived pre-OBs.

\subsubsection{Matrigel assay}

A 96-well cell culture plate chilled at $4^{\circ} \mathrm{C}$ was loaded with $32 \mu$ Matrigel (BD Biosciences, USA) and incubated at $37^{\circ} \mathrm{C}$. Cells were suspended in ENDO medium at a concentration of $2.1 \times 10^{5}$ cells $/ \mathrm{ml}$ and $64 \mu \mathrm{l}$ of this cell suspension was seeded in each well onto the surface of the solidified Matrigel. Then the cells were incubated $\left(37^{\circ} \mathrm{C}\right.$, $5 \% \mathrm{CO}_{2}$ ) for $4 \mathrm{~h}$, after which they were fixed in $10 \% \mathrm{v} / \mathrm{v}$ buffered formalin (Sigma, Germany) for $20 \mathrm{~min}$ at room temperature and further incubated with rhodaminephalloidin (Sigma, Germany) to observe the organization of the cell cytoskeleton. The cell nuclei were counterstained with 4,6-diamidino-2-phenyindole dilactate (DAPI; Invitrogen, USA) for $10 \mathrm{~min}$ and then washed three times with PBS. The samples were visualized using an Axioplan Imager Z1 fluorescence microscope (Zeiss, Germany) and photographed using an Axio Cam MRm camera (Zeiss) running on AxioVision 4.8 software (Zeiss).

\subsubsection{DIL-ac-LDL uptake}

SSEA- $4^{+}$hASC-derived ECs were incubated overnight with $0.2 \mu \mathrm{g} / \mathrm{ml}$ ac-LDL labelled with 1,1'-dioctadecyl-3,3,3',3'tetramethylindocarbocyanine (DIL-ac-LDL, Invitrogen, USA). SSEA-4 ${ }^{+}$hASC-derived pre-OBs were also submitted to the same procedure to confirm the lack of ac-LDL complex uptake ability. The cells were then fixed with $10 \% \mathrm{v} / \mathrm{v}$ buffered formalin for $20 \mathrm{~min}$ at room temperature, protected from light. The cell nuclei were counterstained with DAPI and the samples were further visualized as mentioned previously.

\subsection{Cell number: dsDNA quantification}

The total amount of double-stranded DNA (dsDNA) in each of the mono- and co-culture groups was determined using a fluorimetric dsDNA quantification kit (PicoGreen, Molecular Probes, Invitrogen, USA), according to the manufacturer's instructions. At preselected time points (days 4, 7, 14 and 21 of culture), cells were lysed by osmotic and thermal shocks. Fluorescence was measured using an excitation wavelength of $480 \mathrm{~nm}$ and emission wavelength of $538 \mathrm{~nm}$ in a microplate reader (Synergy HT, Biotek Instruments, USA). Standards were prepared with concentrations in the range $0-2 \mathrm{mg} / \mathrm{ml}$.

\subsection{Metabolic activity assay}

Cellular metabolic activity during co-culture was measured using the CellTiter $®$ Proliferation Assay (MTS; Promega, USA), according to the manufacturer's instructions. Briefly, a MTS working solution was prepared in a 1:5 v/v ratio with phenol red- and serum-free Dulbecco's modified Eagle's medium (DMEM; Gibco, USA). At preselected time points (days 4, 7, 14 and 21), cells were washed thoroughly with PBS and $50 \mu$ MTS working solution were added to each well. The plates were incubated for $2 \mathrm{~h}\left(37^{\circ} \mathrm{C}, 5 \% \mathrm{CO}_{2}\right)$, protected from light, after which optical density (OD) at $490 \mathrm{~nm}$ was measured. The metabolic activity was normalized against the total amount of dsDNA determined for the respective test condition.

\subsection{Alkaline phosphatase activity qualitative and quantitative analysis}

Alkaline phosphatase (ALP) activity was measured using an end-point colorimetric procedure, following an adapted $p$-nitrophenol ( $p N P$ ) assay, on the same cell lysates used for dsDNA quantification. Briefly, $20 \mu$ l samples were incubated with $80 \mu \mathrm{l} 0.2 \% \mathrm{w} / \mathrm{v} p$-nitrophenyl phosphate solution ( $p N P P$ ) in $1 \mathrm{M}$ diethanolamine (Fluka BioChemika, Austria). The OD of the experimental samples and of the calibration curve, prepared using $p$-nitrophenol standards (Sigma, Germany), with values in the range $0-1 \mu \mathrm{M} / \mathrm{ml}$, were read at $405 \mathrm{~nm}$. For the monocultures, ALP activity was normalized against total dsDNA. In the co-cultures, values were normalized against the amount of dsDNA of the CD31 ${ }^{-}$cells ( $\mu \mathrm{M} p \mathrm{NP} / \mathrm{h} / \mu \mathrm{g}$ dsDNA CD31- cells) in the corresponding sample, with the assumption that ALP activity detected for the SSEA- $4^{+}$hASC-derived ECs monocultures is neglectable. ALP activity was represented as the amount of $p$ NP obtained in $1 \mathrm{~h}$ in each cell. The ALP 
activity was also qualitatively assessed by staining the fixed samples with nitro-blue tetrazolium/5-bromo-4chloro-3-indolyl-phosphate (NBT/BCIP; Thermo Scientific, USA). Images were acquired as mentioned above.

\subsection{Real-time reverse transcriptase-polymerase chain reaction (RT-PCR)}

\subsubsection{RNA extraction and CDNA production}

mRNA of the mono- and co-culture samples was extracted using TRI Reagent ${ }^{\circledR}$ (Sigma, Germany), following the manufacturer's instructions. Proteins were extracted using $160 \mu \mathrm{l}$ chloroform (Sigma) and the RNA pellets were washed with an equal volume of isopropanol (Sigma) and $70 \%$ ethanol. The total mRNA was reconstituted in $12 \mu \mathrm{l}$ RNAse/DNAse-free water (Gibco) and its quantity and purity were assessed using a ND1000 Spectrophotometer (NanoDrop Technologies, USA). Samples with a 260:280 ratio in the range 1.6-2.0 were used for the synthesis of single-strand complementary DNA (cDNA). The synthesis of cDNA was performed using qScript ${ }^{\mathrm{TM}}$ cDNA synthesis Kit (Quanta BIosciences, USA) and the thermoblock of the Mastercycler EP Realplex thermal cycler (Eppendorf, USA). An initial amount of $1 \mu \mathrm{g}$ mRNA was used in a total volume of $20 \mu \mathrm{l}$.

\subsubsection{Quantitative real-time $R T-P C R$}

Quantification of the transcripts of the genes of interest was carried out by reverse transcriptase-polymerase chain reaction (RT-PCR), using $50 \mathrm{ng}$ cDNA and PerfeCTA $^{\mathrm{TM}}$ SYBR $\AA$ Green Fast Mix Kit (Quanta Biosciences, USA), following the procedure suggested by the manufacturer. Primers for the genes of interest (Table 2) were previously designed using v. 0.4.0 Primer 3 online software (Whitehead Institute, USA) and synthesized by MGW Biotech (Germany). For each sample, the transcripts' expression data were normalized against the glyceraldehyde-3phosphate-dehydrogenase $(G A P D H)$ housekeeping gene. A concentration of $100 \mathrm{nM}$ each primer was used in a final volume of $20 \mu \mathrm{l}$ sample.

Each real-time RT-PCR reaction was carried out with an initial incubation at $95^{\circ} \mathrm{C}$ for $2 \mathrm{~min}$, followed by 45 cycles of denaturation $\left(95^{\circ} \mathrm{C}, 10 \mathrm{~s}\right)$, annealing (specific for each gene, $30 \mathrm{~s})$ and extension $\left(72^{\circ} \mathrm{C}, 30 \mathrm{~s}\right)$ in a Mastercycler EP Realplex Thermal Cycler (Eppendorf,
USA). The absolute transcript expression data were normalized against endogenous GAPDH values. The relative quantification of targeted genes expression, CD31, vWF, $O C N$ and $O P N$, was performed using the $2^{- \text {delta][delta]Ct }}$ method (Livak and Schmittgen, 2001; Pfaffl, 2001). The CD31 and $v W F$ expression values were normalized against the SSEA- ${ }^{+}$hASC-derived ECs monoculture (100:0) in ENDO and against the number of $\mathrm{CD} 1^{+}$cells obtained from the flow-cytometry data for each condition. The $O C N$ and $O P N$ expression values were normalized against the SSEA- ${ }^{+}$hASC-derived pre-OB monoculture in OST $(0: 100)$ and the number of $\mathrm{CD} 31^{-}$cells for each condition. The normalization was performed for each time point with the corresponding control samples.

\subsection{Immunocytochemistry}

At day 21, cells were fixed with $10 \%$ formalin for $30 \mathrm{~min}$, washed with PBS and incubated with a $3 \% \mathrm{w} / \mathrm{v}$ BSA/PBS solution to block non-specific binding. The cells were incubated for $1 \mathrm{~h}$ at room temperature with mouse antihuman osteocalcin (OCN, 1:50; AbD Serotec, UK) or rabbit anti-human osteopontin (OPN, 1:50; Abcam, Cambridge, UK) primary antibodies diluted in $1.5 \% \mathrm{w} / \mathrm{v}$ BSA/PBS. The samples were then washed with PBS and incubated for $1 \mathrm{~h}$ with the appropriate secondary antibody, either donkey anti-mouse AlexaFluor 488 (Invitrogen, Carlsbad, CA, USA) or donkey anti-rabbit AlexaFluor 488 (Invitrogen), diluted 1:500 in 1.5\% w/v BSA/PBS; the cell nuclei were counterstained with DAPI. Negative control samples were prepared by replacing the primary antibody incubation with PBS. Prior to fixation, the cells were incubated with DIL-ac-LDL, as described above, to allow the localization of SSEA- $4^{+}$hASC-derived ECs by microscopic analysis within the produced extracellular matrix.

\subsection{ELISA}

Secretion of vascular endothelial growth factor (VEGF) was determined by enzyme-linked immunosorbent assay (ELISA) in the supernatants of the co-cultures between days 14 and 21 . The supernatant was centrifuged and filtered before storing at $-80^{\circ} \mathrm{C}$. The amount of human VEGF was quantified following the instructions provided in the ELISA development kit (PeproTech, USA).

Table 2. Primer pair sequences for the genes studied

\begin{tabular}{|c|c|c|c|}
\hline \multirow[b]{2}{*}{ Gene } & \multicolumn{2}{|c|}{ Sequences } & \multirow[t]{2}{*}{$\mathrm{NCBI}$ reference } \\
\hline & Forward (5' $\left.\rightarrow 3^{\prime}\right)$ & Reverse $\left(3^{\prime} \rightarrow 5^{\prime}\right)$ & \\
\hline GAPDH & ACAGTCAGCCGCATC & GACAAGCTTCCCGTTCTCAG & NM_002046.4 \\
\hline CD31 & AAGGCCAGATGCACATCC & TTCTACCCAACATTAACTTAGCGG & NM_000442 \\
\hline$v W F$ & СCCTGGGTTACAAGGAAGAAAT & AGTGTCATGTGTCCTCCTCTAG & NM־ 000552 \\
\hline OPN & GGGGACAACTGGAGTGAAAA & CCCACAGACCCTTCCAAGTA & NM-001040058 \\
\hline OCN & CTGGAGAGGAGCAGAACTGG & GGCAGCGAGGTAGTGAAGAG & NM_099173 \\
\hline
\end{tabular}


Absorbance was read in a multiwell microplate reader at 405 and $650 \mathrm{~nm}$. Standard samples in a range of concentrations of $0-1 \mathrm{ng} / \mathrm{ml}$ recombinant human VEGF were used to obtain a calibration curve.

\subsection{Calcium content quantification}

Detection of inorganic calcium was performed at the end time point of the co-cultures (21 days), using a colorimetric detection kit (Roche, Switzerland) according to the manufacturer's instructions. Briefly, samples were dissolved in a $6 \mathrm{M} \mathrm{HCl}$ solution and transferred to microcentrifuge tubes. In a 96-well plate, $175 \mu 11 \mathrm{M}$ ethanolamine, $\mathrm{pH}$ 10.6, was mixed with $10 \mu \mathrm{l}$ each sample and incubated for $5 \mathrm{~min}$ at room temperature. $70 \mu 10.3 \mathrm{mM} \mathrm{o-cresolphthalein} \mathrm{complex,}$ $13.8 \mathrm{~mm}$ hydroxy-8-quinoline and $122 \mathrm{~mm} \mathrm{HCl}$ solution was added in each well and incubated at $37^{\circ} \mathrm{C}$ for $2 \mathrm{~min}$. The ODs of the samples were read at $570 \mathrm{~nm}$ and the calcium concentrations were extrapolated from the calibration curve obtained using serial dilutions of a $200 \mu \mathrm{g} / \mathrm{ml} \mathrm{CaCl}_{2}$ solution.

\subsection{Statistical analysis}

Statistical analysis was performed using GraphPad Prism 5.00 software (San Diego, CA, USA). The Shapiro-Wilks test was used to ascertain data normality. The results indicated that the non-parametric test should be employed for all comparisons. Statistical analysis of the data were carried using one-way analysis of variance (ANOVA), followed by post hoc Tukey test for all pairwise mean comparisons. Significance levels were set at $p<0.05$.

\section{Results}

\subsection{The endothelial phenotype and an osteogenic precommitment of the cells prior to co-cultures setting confirmation}

The phenotype of SSEA- $4^{+}$hASCs subpopulation after endothelial differentiation and osteogenic preconditioning was analysed prior to setting the co-culture (Figure 1). Endothelial differentiated SSEA-4 ${ }^{+}$hASCs (SSEA-4 ${ }^{+}$ hASC-derived ECs) were characterized by the coexpression of CD31 (94.6\%), CD34 (98.6\%), CD73 (97.8\%) and CD105 (99.7\%), while lacking CD90 (3\%) (Figure 1A). The osteogenic pre-conditioned SSEA-4 ${ }^{+}$ hASCs (SSEA-4 ${ }^{+}$hASC-derived pre-OBs) exhibited a surface marker signature (>98\% CD31 ${ }^{-} / \mathrm{CD} 34^{-} / \mathrm{CD}^{2} 3^{+} /$ $\mathrm{CD}^{+} 0^{+} / \mathrm{CD} 105^{+}$) compatible with a mesenchymal phenotype (Figure 1B) (Zimmerlin et al., 2013). Morphologically, SSEA-4 ${ }^{+}$hASC-derived ECs were characterized by a cobblestone-like appearance (Figure 1C1), while SSEA-4 ${ }^{+}$ hASC-derived pre-OBs displayed an elongated, spindlelike morphology (Figure 1C2), again characteristic of mesenchymal stem cells and similar to the morphology of SSEA $-4^{+}$hASCs cultured in basal conditions ( $\alpha \mathrm{MEM}$ ) (Mihaila et al., 2013).

The SSEA- ${ }^{+}$hASC-derived ECs phenotype was also confirmed by their discriminative ability to uptake the ac-LDL complex (Figure 1D1), as well as to form capillary-like networks when seeded on Matrigel (Figure 1E1). In contrast, SSEA-4 ${ }^{+}$hASC-derived preOBs were not able to uptake ac-LDL (Figure 1D2), and on Matrigel formed aggregates (Figure 1E2). Moreover, the levels of endothelial-specific transcripts CD31 and $\nu W F$ showed a 1500- and 5000-fold increase, respectively, in comparison with the levels displayed by undifferentiated SSEA-4 ${ }^{+}$hASCs prior to osteogenic commitment (Figure 1F). We also confirmed that osteogenic precommitment of SSEA $-4^{+}$hASCs does not alter the constitutive CD31 and $\nu W F$ expression detected prior to conditioning.

The commitment of the SSEA- $4^{+}$hASC-derived preOBs towards the osteogenic lineage was evaluated by considering the activity of ALP, an early marker of differentiation, and the variation of the gene expression profiles of $O P N$ and $O C N$, intermediate and late osteogenicspecific markers, respectively. SSEA- $4^{+}$hASC-derived pre-OBs, SSEA- $4^{+}$hASCs cultured for 7 days in osteogenic medium, depicted significantly higher ALP activity $(p<0.001)$ than uncommitted cells (SSEA-4 ${ }^{+}$hASCs cultured in basal medium) and SSEA- $4^{+}$hASC-derived ECs (Figure 1G). Moreover, a 2.5-fold upregulation $(p<0.05)$ was detected in the expression of $O P N$, while the levels of $O C N$ transcripts did not vary in relation to the uncommitted cells (Figure 1H).

\subsection{Co-culture growth and cellular metabolism dependence with cell ratio and culture media}

The behaviour of SSEA-4 ${ }^{+}$hASC-derived ECs and SSEA$4^{+}$hASC-derived pre-OBs during the co-culture was dependent on both the cell ratio and the culture medium. At initial time points it was possible to discern the spindle-like morphology of SSEA- $4^{+}$hASC-derived preOBs and the cobblestone-like aspect of the SSEA-4 ${ }^{+}$ hASC-derived ECs, independently of the initial cell ratio. For longer time points, fibroblast-like cells took over the culture, forming a dense cellular layer (see supporting information, Figure S1A). The discriminative ability of SSEA-4 ${ }^{+}$hASC-derived ECs to uptake ac-LDL was used to visualize the presence and arrangement of SSEA-4 ${ }^{+}$ hASC-derived ECs during the culture time (Figure 2A, B). Stable SSEA- $4^{+}$hASC-derived ECs monocultures were maintained in both ENDO and MIX media until the end of culture. However, OST medium was not able to support SSEA-4 ${ }^{+}$hASC-derived ECs monocultures, although small DIL-ac-LDL ${ }^{+}$colonies were identified at day 21 in the 75:25 and 50:50 co-cultures (Figure 2B). Thus, although SSEA-4 ${ }^{+}$hASC-derived ECs colonies surrounded by DIL-ac-LDL ${ }^{-}$cells (SSEA-4 ${ }^{+}$hASC-derived pre-OBs) were observed in all the co-cultures, fewer and smaller DILac-LDL ${ }^{+}$colonies were identified with the decrease of SSEA- ${ }^{+}$hASC-derived ECs number. 

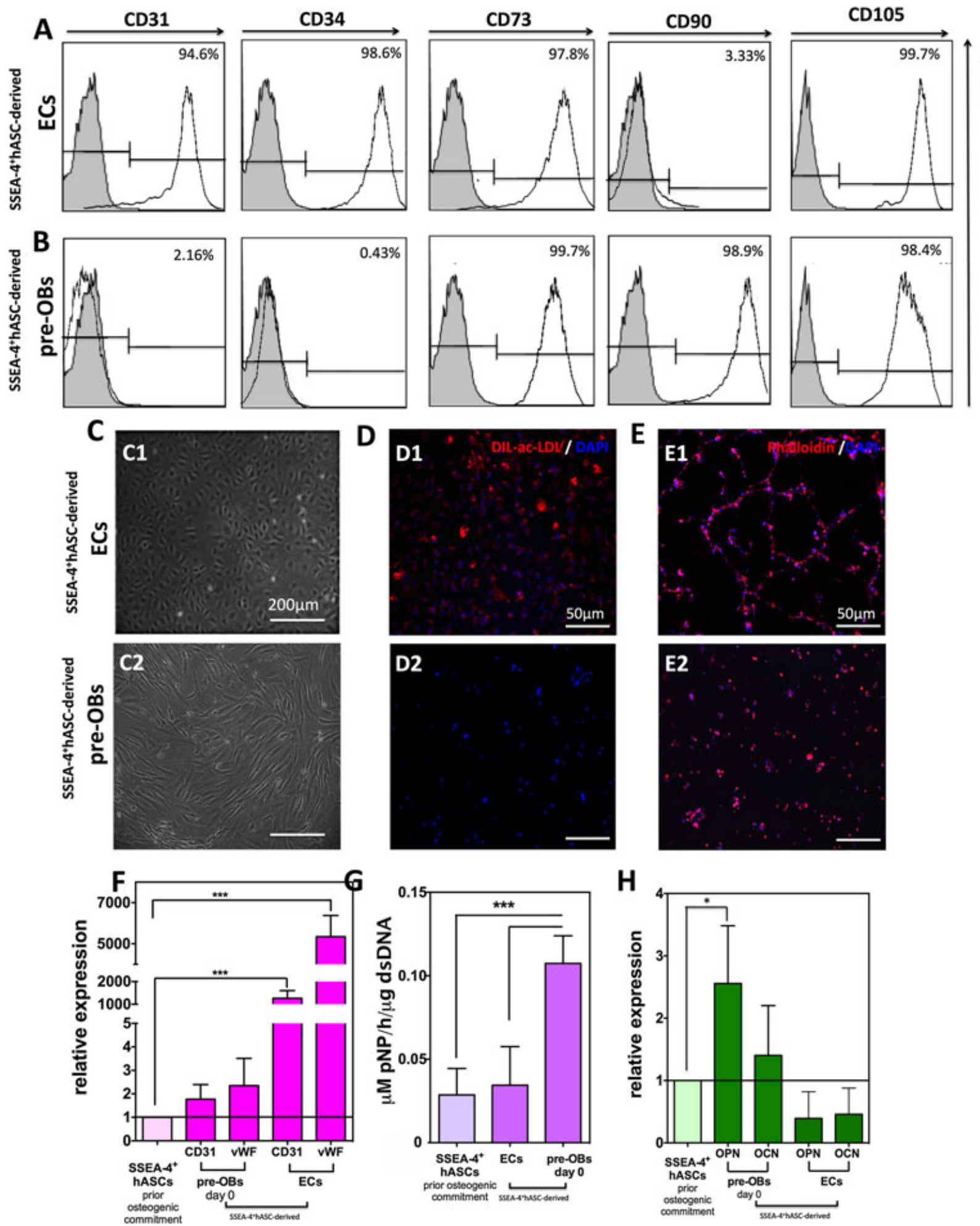

Figure 1. Characterization of endothelial differentiated SSEA-4 ${ }^{+}$hASCs (SSEA- $4^{+}$hASC-derived ECs) and precommitted osteogenic SSEA-4 ${ }^{+}$hASCs (SSEA- $4^{+}$hASC-derived pre-OBs): (A) ECs exhibit a $>94 \% \mathrm{CD}^{+} 1^{+} / \mathrm{CD}^{+} 4^{+} / \mathrm{CD}^{+} 3^{+} / \mathrm{CD}^{-} 0^{-} / \mathrm{CD} 105^{+}$phenotype, while (B) pre-OBs possess a typical mesenchymal surface marker pattern, $>98 \% \mathrm{CD} 31^{-} / \mathrm{CD}^{-} 4^{-} / \mathrm{CD} 105^{+} / \mathrm{CD}^{+} 3^{+} / \mathrm{CD}^{+} 0^{+}$. The two cell types can be discriminated based on their characteristic morphology: (C1) SSEA- $4^{+}$hASC-derived ECs possess a cobblestone-like shape, whereas (C2) SSEA- $4^{+}$hASC-derived pre-OBs are characterized by an elongated, fibroblast-like morphology. (D1) SSEA-4 ${ }^{+}$hASCderived ECs can also be discerned by their ability to uptake the ac-LDL complex, while (D2) SSEA-4 ${ }^{+}$hASC-derived pre-OBs lack this behaviour. (E1) SSEA-4 ${ }^{+}$hASC-derived ECs have the ability to form tubular-like networks when seeded on Matrigel; (E2) in contrast, SSEA- $4^{+}$hASC-derived pre-OBs remain spherical and form aggregates without any evidence of network formation. (F) The levels of endothelial-specific transcripts CD31 and $v W F$ in SSEA $-4^{+}$hASC-derived ECs were significantly higher than those displayed by the undifferentiated SSEA-4 ${ }^{+}$hASCs. (G) The commitment of the SSEA- $4^{+}$hASC-derived pre-OBs towards the osteogenic lineage was confirmed by increased activity of alkaline phosphatase and $(\mathrm{H})$ a significant upregulation of OPN transcripts in relation to undifferentiated SSEA- $4^{+}$hASCs; no upregulation of $O C N$ was observed for SSEA- $4^{+}$hASC-derived pre-OBs. Data are expressed as mean \pm standard deviation (SD); $n=3 ;{ }^{*} p<0.05, * * * p<0.001$ 
A

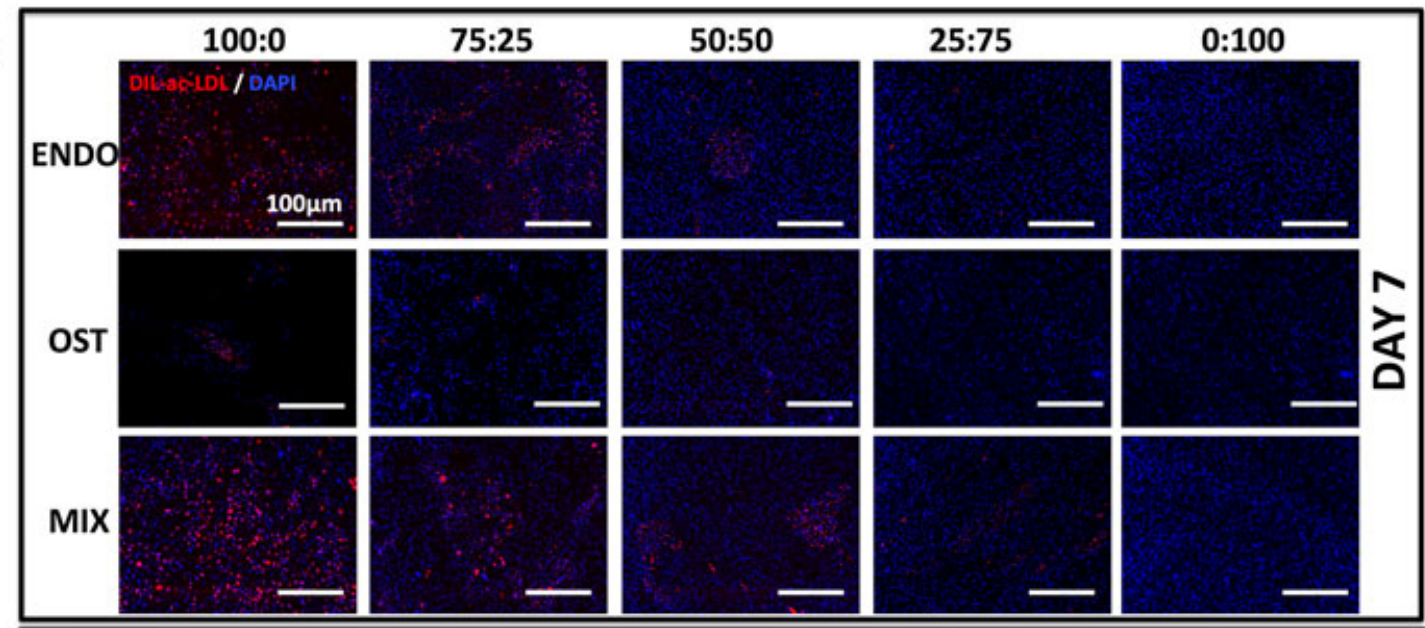

B

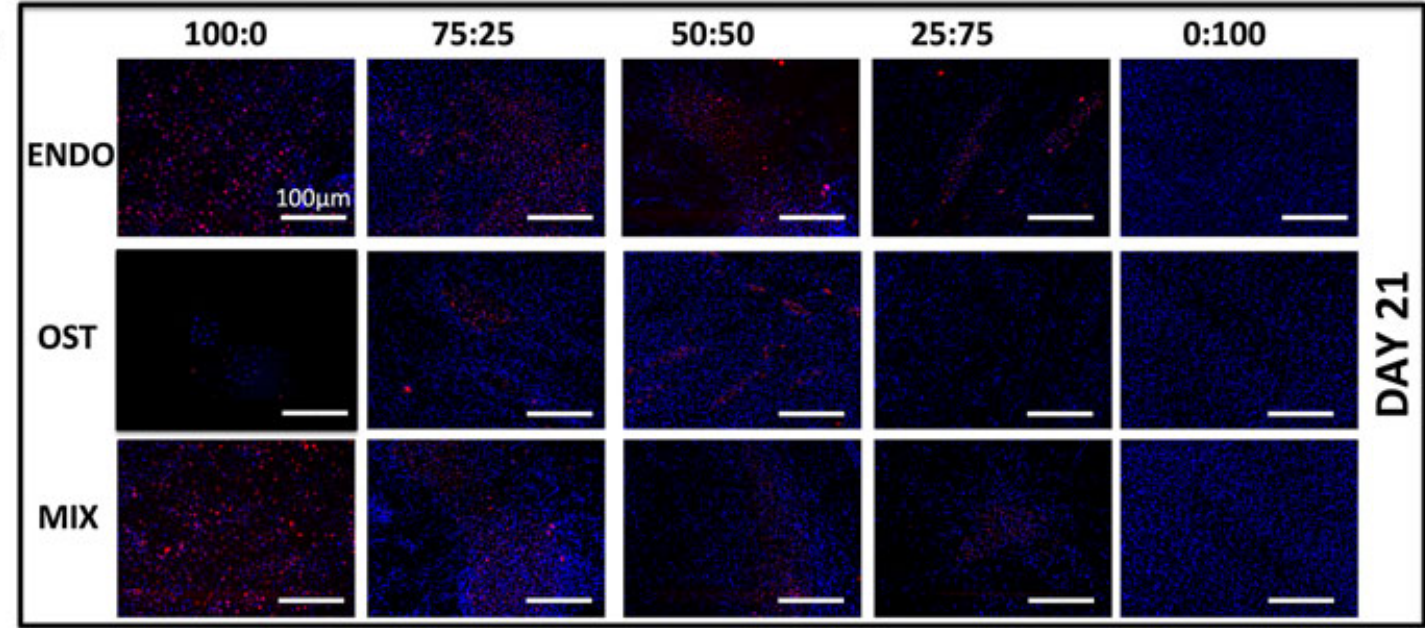

C
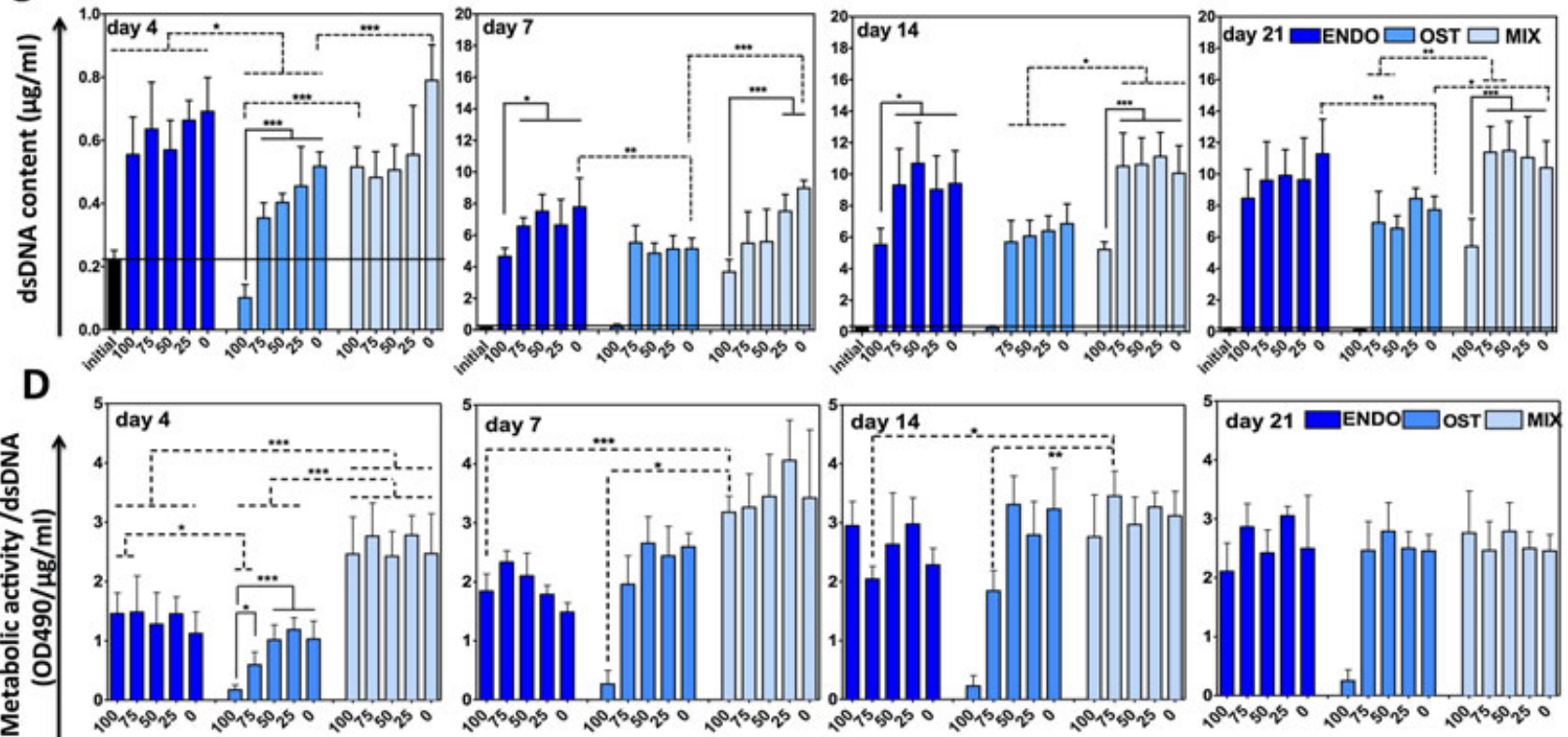

Percentage SSEA-4'hASC-derived ECs (\%) and time (days)

Figure 2. Evaluation of cell organization and proliferation through culture time. (A, B) ECs were distinguished from pre-OBs by their specific ability to uptake ac-LDL complex (red) and were found arranged in small colonies amongst DIL-ac-LDL cells in all the co-cultures, although fewer and smaller colonies were identified with decreasing SSEA-4 ${ }^{+}$hASC-derived ECs ratio. Stable SSEA-4 ${ }^{+}$ hASC-derived ECs monocultures were maintained in both ENDO and MIX, but not in OST media; cell nuclei were counterstained with DAPI (blue). (C) Cell proliferation, traduced by dsDNA content through time, shows strong dependence on the cell ratio and culture media. (D) Cellular metabolism was influenced by the culture media, but only at early time points; with time, only the metabolic activity of SSEA- ${ }^{+}$hASC-derived ECs in monoculture in OST medium was significantly lower than in the other two media. Data are expressed as mean $\pm \mathrm{SD} ; n=3 ; * p<0.05, * * p<0.01$, $* * * * 0.001$ 
The cell proliferation results confirmed the dependence of cellular growth on the cell ratio and culture medium (Figure 2C). In general, all the co-cultures exhibited significant proliferation up to day $14(p<0.05)$. SSEA-4 $4^{+}$ hASC-derived pre-OB monocultures in ENDO and MIX medium exhibited the highest growth rates, and a significantly lower growth $(p<0.05)$ in OST. This trend was also observed for the co-cultures in the respective media. The dsDNA quantification also corroborated the morphological analysis of the cultures, showing that SSEA-4 ${ }^{+}$ hASC-derived ECs have a significantly lower $(p<0.05)$ proliferation rate than SSEA- $4^{+}$hASC-derived pre-OBs mono- and co-cultures, in both ENDO and MIX media, and that OST medium does not support SSEA-4 ${ }^{+}$hASCderived ECs growth.

The metabolic activity results showed that, for the earliest time point and independently of the ratio, cells were significantly more active $(p<0.001)$ in MIX than ENDO medium. However, differences were detected at day 14 for the 75:25 ratio in MIX in comparison to the same ratio in ENDO and OST. Moreover, the metabolic activity of SSEA-4 ${ }^{+}$hASC-derived ECs monocultures in OST medium was significantly lower than in the other two media, and in the other cell ratios in the same medium (Figure 2D). Thus, cellular metabolism was mainly influenced by the culture medium and predominantly at early time points.

\subsection{Evolution of endothelial phenotype along the culture}

To clearly follow the behaviour of SSEA- $4^{+}$hASC-derived ECs during the culture, the percentage of $\mathrm{CD} 31^{+}$in the co-cultures was determined by flow cytometry. The CD $31^{-}$profile of SSEA- $4^{+}$hASC-derived pre-OB monocultures was confirmed by the lack of CD31 expression during the 21 days of culture, regardless of the culture medium. In contrast, $>95 \%$ of the ECs in monocultures in ENDO and MIX media expressed CD31, which was maintained until the end of culture (Figure 3A). The percentage of $\mathrm{CD}_{3} 1^{+}$cells in each of the cell ratios did not vary between the ENDO and MIX media for the entire duration of the culture, except for the 75:25 cell ratio at day 21 in MIX, in which the percentage of SSEA- $4^{+}$hASCderived ECs cells was significantly higher $(p<0.001)$ than in the ENDO and OST media. Nonetheless, neither ENDO nor MIX media were capable of preserving the initial cell ratio. Even more, the percentage of $\mathrm{CD} 31^{+}$cells in OST medium was always significantly lower than that in the other two media up to 7 days for all the defined cell ratios. However, at day 14 for the 25:75 cell ratio and at day 21 for the 50:50 and 25:75 cell ratios, the percentage of $\mathrm{CD} 31^{+}$cells in the three media was similar.

In addition to evolution in terms of growth, the stability of the endothelial phenotype of SSEA- $4^{+}$hASC-derived ECs in co-culture was evaluated by analysis of the expression of the genes CD31 and $v W F$ (Figure 3B, C) in relation to the SSEA-4 ${ }^{+}$hASC-derived ECs monocultures in ENDO medium, which showed stable expression of both CD31 and $v W F$ during 21 days of culture (see supporting information, Figure S2A). We also confirmed that the SSEA$4^{+}$hASC-derived pre-OB monocultures did not express $C D 31$ or $v W F$ in any of the three culture media, maintaining this neglectable expression profile along the culture (see supporting information, Figure S2B). While the expression levels of $C D 31$ and $v W F$ were not affected by the culture in MIX and ENDO media, the SSEA-4 ${ }^{+}$ hASC-derived ECs phenotype was altered by the culture in OST medium. A downregulation of both genes was observed in the SSEA- ${ }^{+}$hASC-derived ECs monocultures up to 21 days of culture. However, in all the other co-culture conditions, the expression of CD31 and $v W F$ was stable.

\subsection{Evolution of osteogenic differentiation during the culture}

To analyse the progress of the SSEA- $4^{+}$hASC-derived preOBs during the culture time and under the various study conditions, a panel of markers associated with the different stages of osteogenic differentiation was assessed. The quantification of ALP activity of the cultures in ENDO medium did not reveal variations with time in relation to the initial SSEA- $4^{+}$hASC-derived pre-OBs value for any of the cell ratios, including the pre-OB monocultures (Figure 4A). In opposition, the typical profile of ALP activity associated with osteogenic differentiation was observed for all the co-cultures and the SSEA- $4^{+}$hASCderived pre-OB monocultures in both OST and MIX media. An activity peak was reached at day 7, with significantly higher $(p<0.001)$ values than the constitutive activity and the corresponding groups in ENDO medium. Interestingly, the ALP activity detected for the 75:25 cell ratio in MIX medium was significantly higher $(p<0.05)$ than for the pre-OB monocultures in MIX and OST media.

Quantitative analysis of ALP activity was corroborated by the qualitative results. The constitutive ALP activity of both SSEA-4 ${ }^{+}$hASC-derived ECs and SSEA-4 ${ }^{+}$hASCderived pre-OBs was represented by the staining of randomly dispersed cells in the different ratios in ENDO medium at day 4 (Figure 4B). At the endpoint of the experiment, a slight variation of the staining intensity due to the higher number of cells was detected in ENDO medium, but considerably less intense than for the co-cultures and SSEA-4 ${ }^{+}$hASC-derived pre-OB monocultures in OST and MIX media (Figure 4C).

The osteogenic differentiation of SSEA $-4^{+}$hASCderived pre-OBs in co-cultures was confirmed at the gene level by quantifying expression of the osteogenic-specific genes osteopontin $(O P N)$ and osteocalcin $(O C N)$ in relation to the SSEA-4 ${ }^{+}$hASC-derived pre-OB monoculture in OST (Figure 5A, B) at each corresponding time point. In order to make this comparison, complete differentiation of the SSEA- $4^{+}$hASC-derived pre-OB monoculture in OST was confirmed (see supporting information, Figure S3A1, A2). The expression of $O P N$, an intermediate 

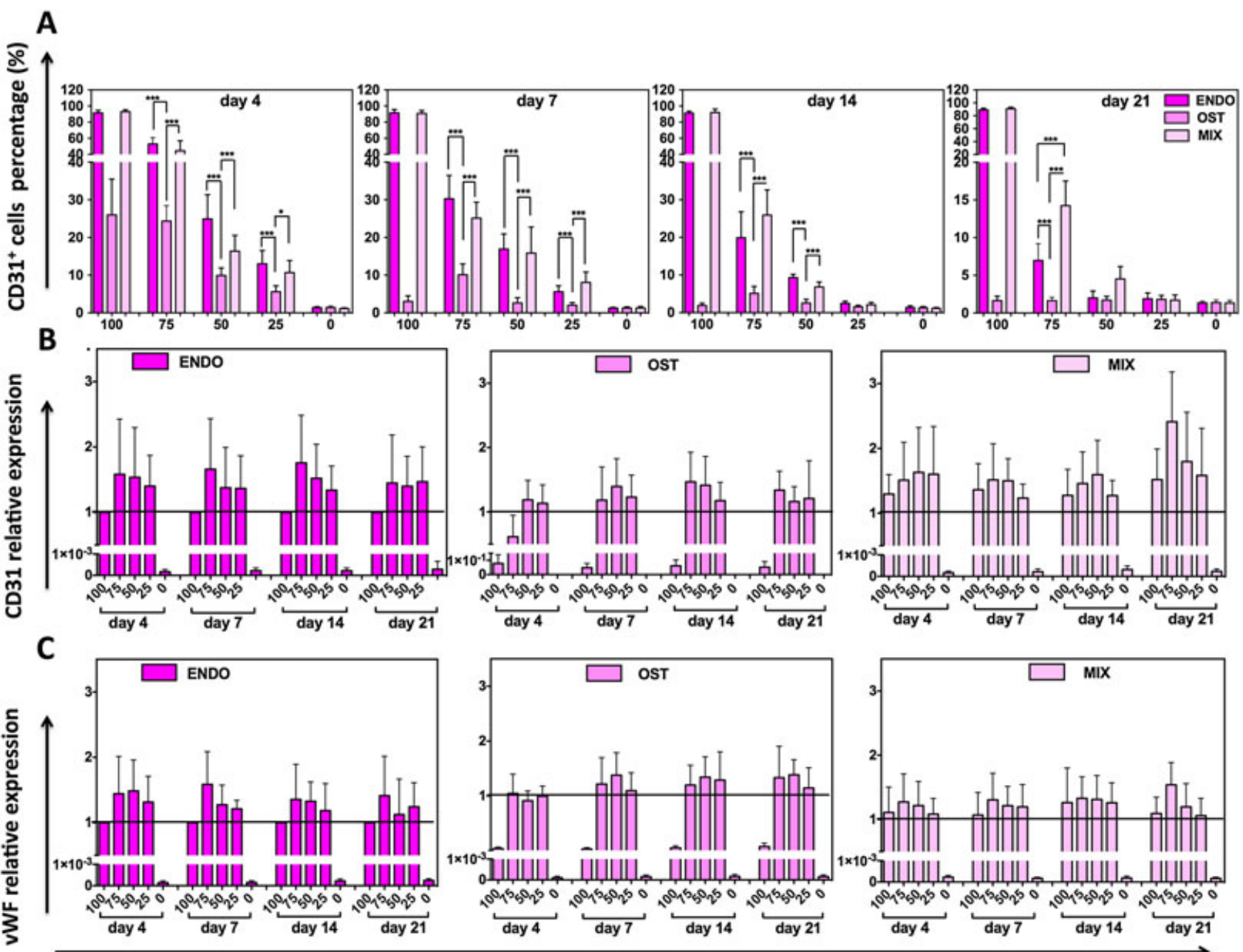

Percentage SSEA-4 ${ }^{+}$hASC-derived ECs (\%) and time (days)

Figure 3. Evolution of endothelial phenotype through culture time. (A) Percentage of $\mathrm{CD}^{+}{ }^{+}$cells determined by flow cytometry. The CD31 ${ }^{-}$profile of SSEA- $4^{+}$hASC-derived pre-OB monocultures was confirmed by their lack of CD31 expression during the 21 days of culture, regardless of the culture medium; in contrast, $>95 \%$ of the SSEA- $4^{+}$hASC-derived ECs monocultures in ENDO and MIX media expressed CD31, which was maintained until the end of culture. Neither ENDO nor MIX media were capable of preserving the initial cell ratio. The stability of the endothelial phenotype of SSEA- $4^{+}$hASC-derived ECs in co-culture was evaluated by analysis of the expression of (B) CD31 and (C) $\nu W F$ genes in relation to the EC monocultures in ENDO medium. While the expression levels of $C D 31$ and $v W F$ were not influenced by MIX medium, the SSEA- $4^{+}$hASC-derived EC phenotype in the monocultures up to 21 days of culture, and in the 75:25 co-cultures at day 4, was affected by OST medium. The relative expression of each gene was normalized against SSEA-4 ${ }^{+}$hASC-derived ECs monocultures in ENDO medium and against the number of CD31 ${ }^{+}$cells obtained from the flow-cytometry data for each condition, with the assumption that only $C D 31^{+}$cells contribute to the $C D 31$ and $v W F$ transcripts. Data are expressed as mean $\pm \mathrm{SD} ; n=3 ;{ }^{*} p<0.05,{ }^{* *} p<0.01, * * p<0.001$

marker of differentiation, significantly $(p<0.001)$ increased until reaching a maximum of 90 -fold at day 7. Consistently, the expression of $O C N$, known as a late marker of differentiation, significantly increased $(p<0.001)$ with time, reaching a 2500 -fold gain at day 21 . Thus, analysis of the expression of $O P N$ and $O C N$ in the co-cultures in OST and MIX media did not show significant differences in relation to the SSEA- $4^{+}$hASC-derived pre-OB monocultures, except for the 75:25 cell ratio in MIX medium. In this condition, the expression of $O P N$ and $O C N$, respectively, at days 7 and 14 was significantly higher $(p<0.01)$ than in the SSEA- $4^{+}$hASC-derived pre-OB monocultures in both OST and MIX media (Figure 5A, B). Additionally, SSEA-4 ${ }^{+}$hASC-derived pre-OB mono- and cocultures in ENDO medium displayed significantly $(p<0.001)$ lower expression of $O P N$ and $O C N$ than the respective reference condition (SSEA-4 ${ }^{+}$hASC-derived pre-OB monoculture in OST) (Figure 5A, B). The gene expression results were confirmed at the protein level. The deposition of an OPN- and OCN-enriched matrix surrounding small ac-LDL ${ }^{+}$colonies was confirmed by immunocytochemistry (Figure 5C).

The ultimate marker of osteogenic differentiation, matrix mineralization, was indirectly assessed by quantification of the amount of inorganic calcium present in culture (Figure 5D) (Amosi et al., 2012). While in the SSEA- $4^{+}$hASC-derived ECs monocultures the residual amount of calcium (cca $0.5 \mu \mathrm{g} / \mu \mathrm{g}$ dsDNA) did not vary with the medium, in the SSEA- $4^{+}$hASC-derived pre$\mathrm{OB}$ monocultures and co-cultures a medium- and 
A

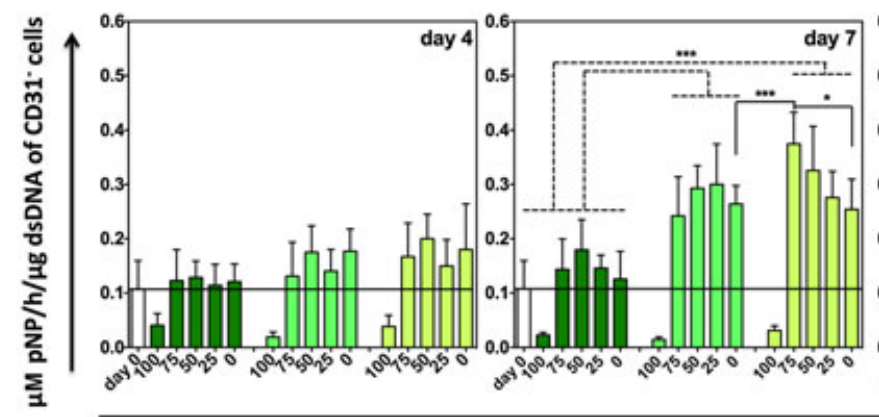

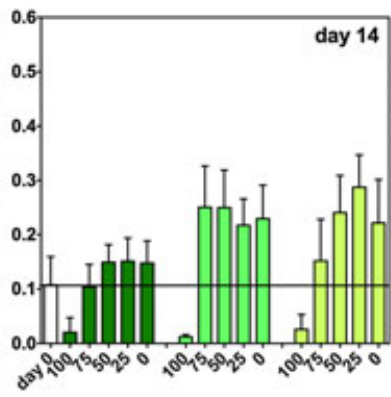

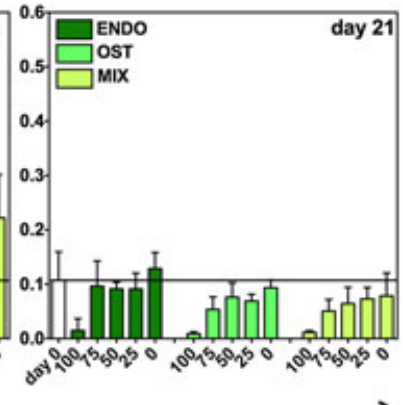

Percentage SSEA-4 ${ }^{\text {h }}$ ASC-derived ECs (\%) and time (days)

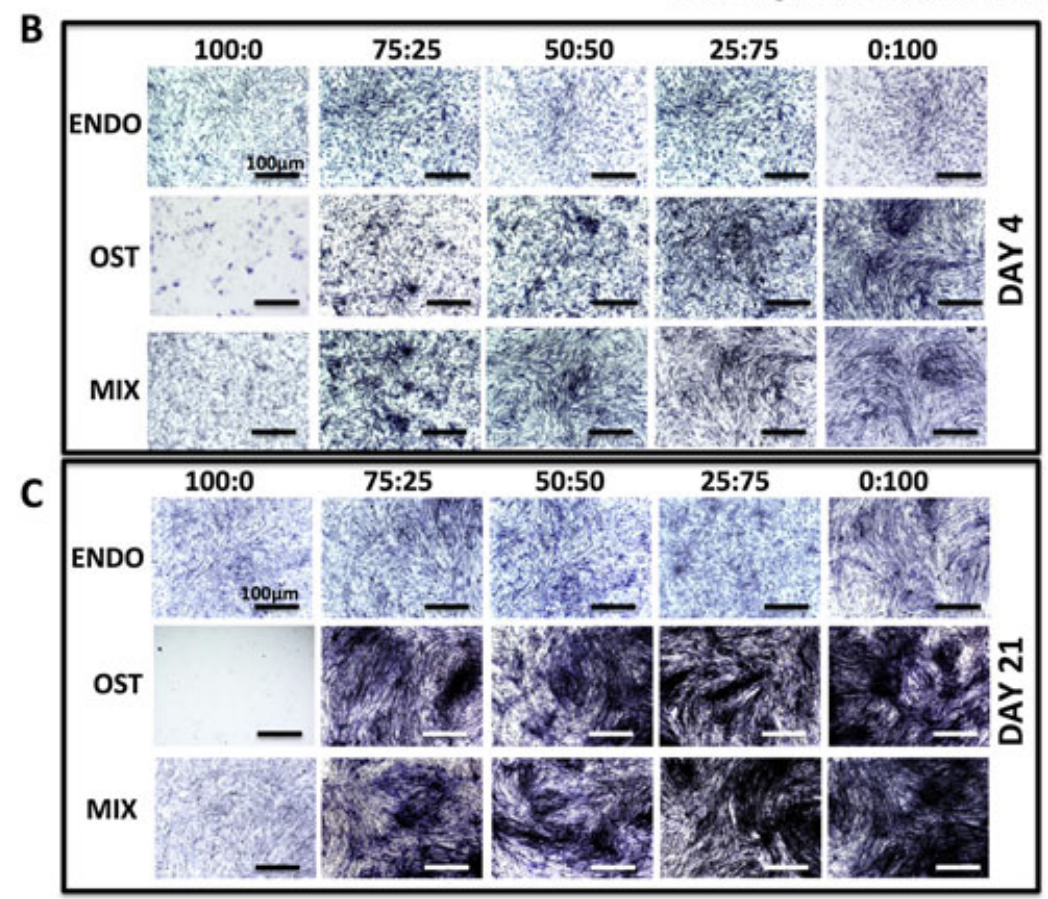

Figure 4. Alkaline phosphatase (ALP) activity through culture time. (A) Quantification of ALP activity of the cultures in ENDO medium did not reveal variations with time in relation to the initial SSEA- $4^{+}$hASC-derived pre-OBs value for any of the cell ratios, including the SSEA- $4^{+}$hASC-derived pre-OB monocultures. Conversely, the typical profile of ALP activity associated with osteogenic differentiation was observed for all the co-cultures and the SSEA- $4^{+}$hASC-derived pre-OB monocultures in both OST and MIX media. Quantitative analysis of ALP activity was corroborated by qualitative results showing: (B) the constitutive ALP activity of both SSEA$4^{+}$hASC-derived ECs and SSEA-4 ${ }^{+}$hASC-derived pre-OBs (purple) under the different conditions in ENDO medium at day 4, with a significantly lower intensity than in the co-cultures and SSEA- $4^{+}$hASC-derived pre-OB monocultures in OST and MIX media; and (C) confirmation of osteogenic differentiation only in OST and MIX at day 21. For the monocultures, ALP activity was normalized against total dsDNA. In the co-cultures, values were normalized against the amount of dsDNA of the CD31 ${ }^{-}$cells $(\mu \mathrm{M}$ pNP/h/ $\mu \mathrm{g}$ dsDNA of $\mathrm{CD}^{-} 1^{-}$cells) in the corresponding sample, with the assumption that ALP activity detected for the SSEA- $4^{+}$hASC-derived ECs monocultures is neglectable. ALP activity is represented as the amount of pNP obtained in $\mathbf{1 ~ h}$ in each cell. Data are expressed as mean \pm $\mathrm{SD} ; n=3 ; * p<0.05,{ }^{* *} p<0.01, * * * p<0.001$

ratio-dependent calcium content was detected. Moreover, the level of calcium found in the SSEA- $4^{+}$hASC-derived ECs monocultures and in the SSEA- $4^{+}$hASC-derived pre-OB monocultures and co-cultures in ENDO medium, was comparable. In opposition, in OST and MIX media the levels of calcium significantly increased $(p<0.001)$. Interestingly, in MIX medium, the amount of calcium detected in the 75:25 co-culture was significantly higher than in all the other conditions in the same medium $(p<0.001)$, and in co-cultures in OST $(p<0.001)$ and SSEA- $4^{+}$hASC-derived pre-OB monoculture $(p<0.001)$ in OST medium, an observation that is valid for the 50:50 ratio in MIX as well. No differences between the 25:75 and pre-OB monocultures in MIX and OST were observed.

\subsection{Medium- and cell ratio-dependent VEGF release}

The amount of VEGF secreted in the different cultures at the end time point was determined in order to confirm a possible VEGF-mediated crosstalk between the endothelial and osteogenic cells under different culture conditions (Figure 6A). In OST conditioned medium, the amount of VEGF did not vary with the cell ratios. In opposition, both the cell ratio and the ENDO and MIX conditioned media affected the release of VEGF. In the 75:25 cellular ratio in ENDO conditioned medium a significantly higher $(p<0.001)$ VEGF amount was detected than in the SSEA- $4^{+}$hASC-derived ECs and SSEA-4 ${ }^{+}$hASC-derived 
A
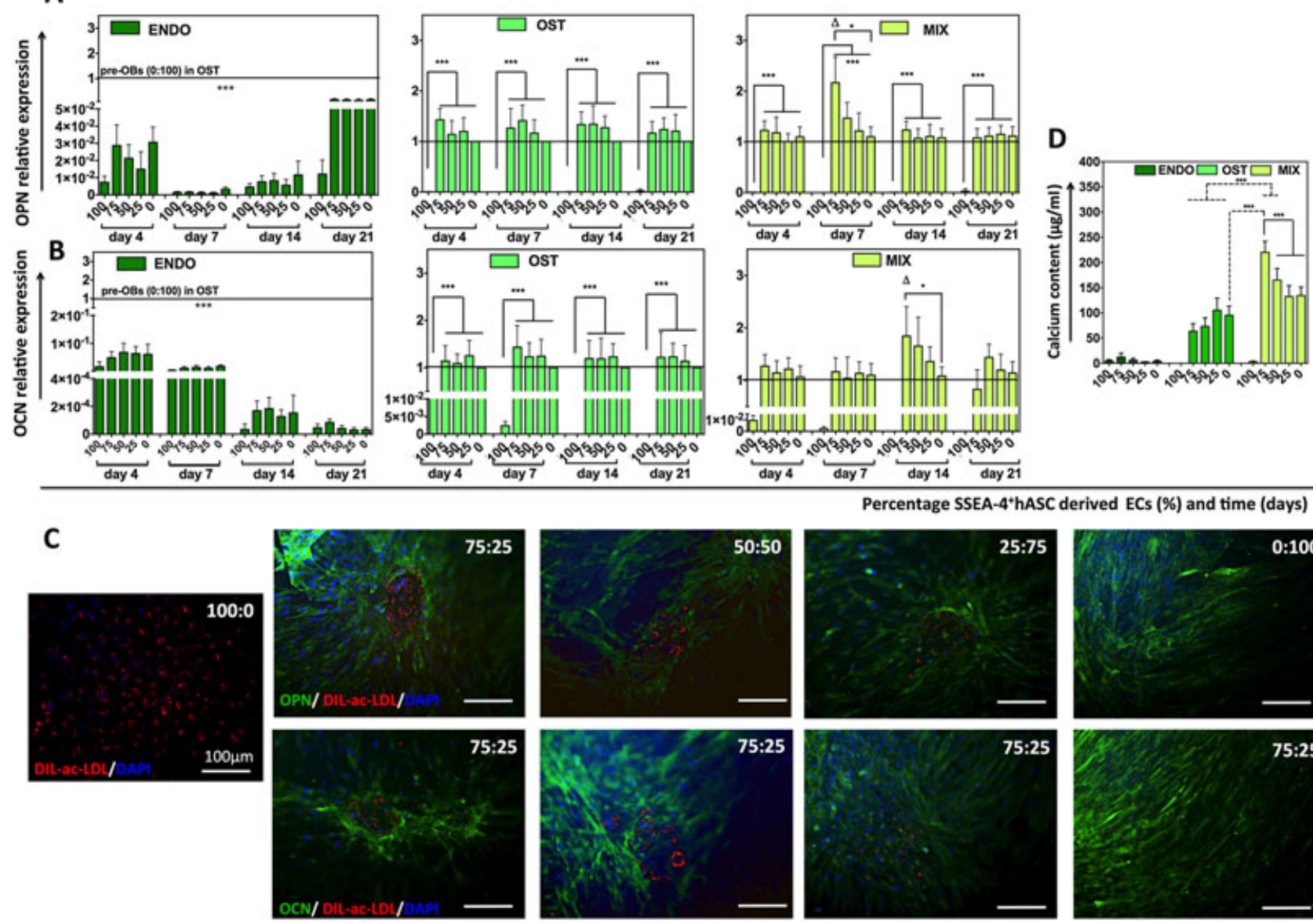

Percentage SSEA-4 hASC derived ECs (\%) and time (days)
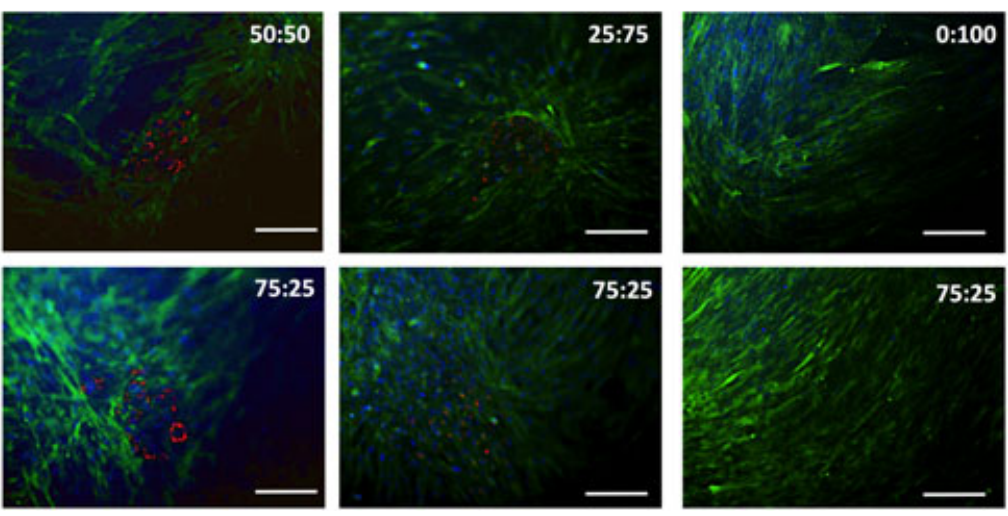

Figure 5. Characterization of osteogenic differentiation through culture time. Osteogenic differentiation of SSEA- $4^{+}$hASC-derived pre-OBs in co-cultures was confirmed by the expression of osteogenic-specific genes, (A) osteopontin (OPN) and (B) osteocalcin (OCN), at the molecular and (C) protein levels, as indicated by the deposition of an OPN- and OCN-enriched matrix surrounding small DIL-ac-LDL ${ }^{+}$colonies. OPN and OCN expression values were normalized against SSEA-4 ${ }^{+}$hASC-derived pre-OB monoculture in OST (0:100) and the number of CD31 ${ }^{-}$cells for each condition. (D) The amount of inorganic calcium deposited in the extracellular matrix, an indirect indicator of mineralization, was evaluated after 21 days of culture for all conditions; the highest levels of calcium were found in the 75:25 ratio. Data are expressed as mean \pm SD; $n=3 ; * p<0.05, * * p<0.01, * * * p<0.001 ; \Delta$, significant difference $(p<0.05)$ from SSEA- $4^{+}$hASC-derived pre-OBs in the OST condition at the same time point
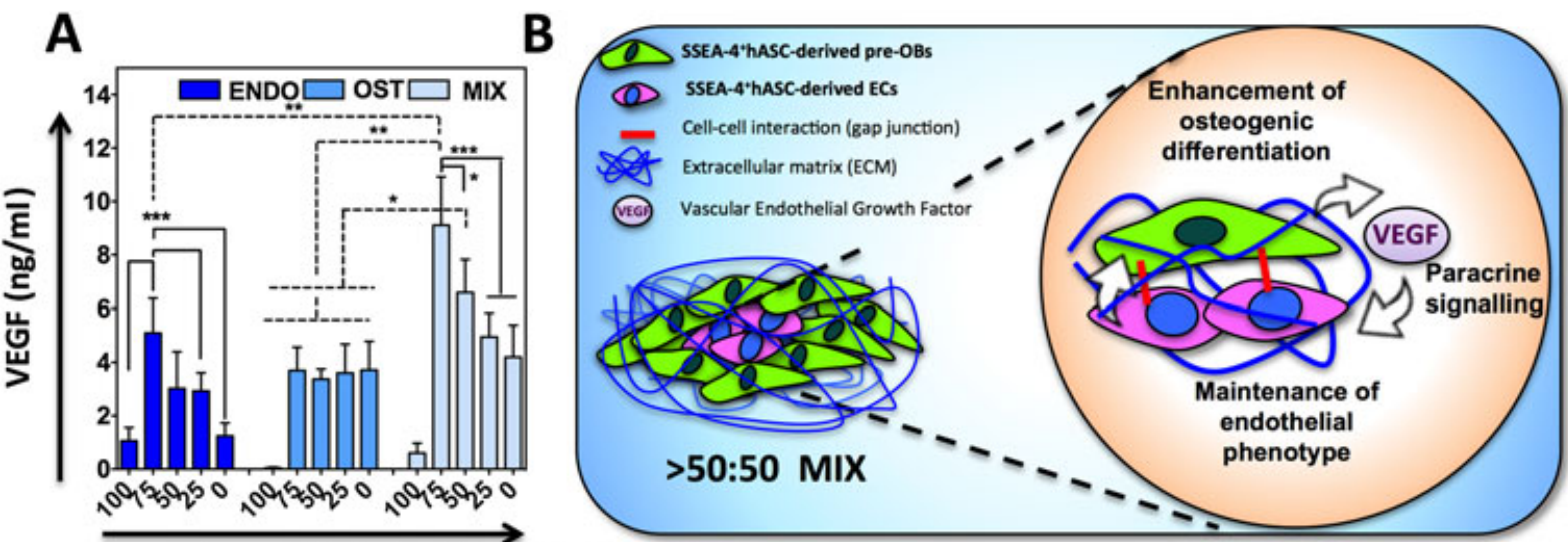

Percentage SSEA-4+hASC-derived ECs (\%)

Figure 6. VEGF-mediated crosstalk between endothelial and osteogenic cells. (A) VEGF secretion quantified in the supernatant of the cultures at the end time point; data are expressed as mean \pm SD; $n=3 ; * p<0.05, * * p<0.01, * * * p<0.001$. (B) Schematic representation of paracrine signalling between differentiated SSEA- $4^{+}$hASC-derived pre-OBs and SSEA- $4^{+}$hASC-derived ECs involved in increased matrix mineralization and maintenance of the endothelial phenotype in the 75:25 MIX condition. It is proposed that the presence of ECs, maintained by the VEGF of the medium, synergizes with the MIX-conditioned medium, enhancing the osteogenic differentiation of SSEA- $4^{+}$hASC-derived pre-OBs. This leads to increased VEGF release by the pre-OBs/differentiated cells sustaining the ECs phenotype and growth and, thus, promoting a VEGF-associated signalling loop 
pre-OB monocultures and in the 25:75 ratio. Nonetheless, that amount of VEGF was significantly lower $(p<0.001)$ than that detected for the same cell ratio (75:25) in MIX conditioned medium $(9.1 \pm 1.8 \mathrm{ng} / \mathrm{ml})$, in turn significantly higher $(p<0.01)$ than in the other conditions in MIX conditioned medium. Notably, the VEGF level for the 75:25 and 50:50 in MIX were significantly higher than in all cultures in OST. Furthermore, the VEGF secretion in the SSEA-4 ${ }^{+}$hASC-derived ECs monocultures in ENDO $(0.61 \pm 0.34 \mathrm{ng} / \mathrm{ml})$ and MIX (0.60 $\pm 0.36 \mathrm{ng} / \mathrm{ml})$ media and in the SSEA- $4^{+}$hASC-derived pre-OB monoculture in ENDO was comparable and significantly lower $(p<0.001)$ than in all the other conditions. Moreover, the VEGF values detected in the SSEA-4 ${ }^{+}$hASC-derived pre-OB cultures in OST and MIX conditioned media were similar.

\section{Discussion}

During bone tissue regeneration, the metabolic requirements of the local environment led to the upregulation of multiple downstream events aimed at promoting vessel ingrowth and supporting osteogenic functionality. These phenomena rely on the intimate cooperation between bone-forming cell precursors and microvascular endothelial cells. There are several studies (Grellier et al., 2009; Pirraco et al., 2010; Valenzuela et al., 2013) on the reciprocal regulation and functional relationship between preOBs and ECs which, in turn, may be greatly influenced by the degree of osteogenic maturation of pre-OBs and culture conditions. Although the beneficial effects seem to be conserved, there is still a lack of consensus regarding the ideal combination of cells and the respective conditions necessary to maximize their potential. This study intended to tackle this issue by determining under which cell ratio and culture conditions SSEA- $4^{+}$-derived preOBs and SSEA- $4^{+}$hASC-derived ECs communicate synergistically to support the full differentiation of SSEA-4 ${ }^{+}$ hASC-derived pre-OBs and the maintenance of the SSEA- $4^{+}$hASC-derived ECs phenotype.

Culture medium has been the most useful tool in in vitro cell culture to guarantee the minimal requirements for cell survival, growth and, eventually, differentiation. We defined ENDO medium, composed by a cocktail of growth factors, amongst which VEGF $[<5 \mathrm{ng} / \mathrm{ml}$ (Ferreira et al., 2007)], which significantly contributes to the maintenance of the endothelial phenotype, and standard osteogenic medium (Porter et al., 2003) (OST), as the ideal conditions for SSEA- ${ }^{+}$hASC-derived ECs and SSEA-4 ${ }^{+}$hASC-derived pre-OBs culture, respectively. Considering our goal, we assumed that by combining the ingredients of the two media (MIX), both cell types would be provided with the required factors at the standard optimum concentrations. Similar approaches have been followed in previous studies (Ma et al., 2011; Valenzuela et al., 2013), although the ENDO and OST media were mixed at a 1:1 ratio, reducing to half the concentrations of the provided factors.
While the selection of the media is somehow consensual, the EC:pre-OBs ratios that have been tested represent a wide range of variations, from 95:5 (Ma et al., 2011), 80:20 (Ma et al., 2011; Santos et al., 2009; Unger et al., 2007), 75:25 (Bidarra et al., 2011), 66:33 (Maritie Grellier et al., 2009; Guillotin et al., 2008; Villars et al., 2002), 50:50 (Hofmann et al., 2008; Kaigler et al., 2005) and 25:75 (Bidarra et al., 2011) down to 2:98 (Ma et al., 2011). A common outcome of these studies refers to the need for a higher percentage of ECs in longterm cultures, as pre-OBs, OBs or even MSCs have a higher proliferation rate and thus overtake ECs and hinder their modulatory behaviour (Unger et al., 2007; Villars et al., 2002) and, consequently, the osteogenic commitment of the overall culture. Based on these conclusions, and considering our previous knowledge regarding the SSEA-4 ${ }^{+}$hASC-derived ECs' proliferation ability, we selected the $75: 25,50: 50$ and $25: 75$ (SSEA-4 ${ }^{+}$hASCderived ECs:SSEA-4 ${ }^{+}$hASC-derived pre-OBs) ratios.

Both cell ratio and culture medium impacted cell growth and the metabolic activity of the co-cultures. Although SSEA-4 ${ }^{+}$hASC-derived ECs did not survive in OST medium, as expected, SSEA- $4^{+}$hASC-derived ECs colonies were found in the 75:25 and 50:50 conditions. Thus, our results showed not only that the presence of the SSEA-4 ${ }^{+}$hASC-derived pre-OBs supports the SSEA$4^{+}$hASC-derived ECs survival but also that an equivalent or higher starting number of SSEA- $4^{+}$hASC-derived ECs allows the maintenance of the co-cultures for up to 21 days. This is in agreement with previous studies (Bidarra et al., 2011; Ma et al., 2011; Xue et al., 2009) that showed the smallest decrease of ECs numbers coupled together with the highest proliferation of differentiating cells in the conditions with the highest numbers of ECs in the starting culture. We were also able to prove that both cell types were metabolically active and highly proliferating in both ENDO and MIX, although with significantly different growth rates, as expected. Interestingly MIX and ENDO media showed similar results in terms of cell proliferation, but the cells were significantly metabolically more active in MIX medium, particularly at early time points. Interestingly, each monoculture displayed a higher initial metabolic activity in MIX than in its own standard culture medium. We suggest that the enriched formulation of the MIX medium potentiates the immediate adjustment of both SSEA-4 ${ }^{+}$hASC-derived ECs and SSEA- $4^{+}$hASCderived pre-OBs, alone or in co-culture, to its components, as they were previously cultured in ENDO and OST, respectively.

In agreement with the conditions that support the survival of SSEA- $4^{+}$hASC-derived ECs during the culture, in ENDO and MIX media SSEA-4 ${ }^{+}$hASC-derived ECs monocultures retained their endothelial signature during the culture period. In OST medium, a downregulation of CD31 and $\nu W F$ was observed only in the SSEA- $4^{+}$hASCderived ECs monocultures. This confirms the loss of the endothelial signature, which is an indication that OST is not a suitable culture medium for this type of cell. Moreover, the percentage of $\mathrm{CD} 31^{+}$cells in the 75:25 cell ratio 
and MIX medium was significantly higher than in ENDO. These results suggest that the supplements present in ENDO medium are essential for the survival, growth and maintenance of the endothelial phenotype, as already reported elsewhere (Bidarra et al., 2011; Ma et al., 2011), but not sufficient to enhance their growth when cultured together with SSEA- $4^{+}$hASC-derived pre-OBs. Moreover, in OST, the presence of SSEA- $4^{+}$hASC-derived pre-OBs is mandatory to maintain the endothelial phenotype, most likely due to their regulatory crosstalk with the SSEA-4 ${ }^{+}$ hASC-derived ECs. In fact, pre-OBs at different stages of differentiation are known to influence ECs' activity by the release of angiogenic factors, among which is VEGF (Deckers et al., 2000; Dohle et al., 2010; Street and Lenehan, 2009), present in both initial formulations of MIX and ENDO media at the same concentration but not in OST.

Interestingly, both ENDO and MIX media, containing the elements that were shown to trigger the endothelial differentiation of freshly isolated SSEA- $4^{+}$hASCs (Mihaila et al., 2013), failed to induce the acquisition of endothelial-like features by SSEA-4 ${ }^{+}$hASC-derived preOBs, which seems to indicate that the preconditioning step blocks their differentiation potential towards the endothelial lineage. This, in addition to the increased values of ALP activity, an early hallmark of osteogenic differentiation and a prerequisite for the up-front events involving osteogenic matrix deposition and consequent mineralization (Marom et al., 2005), and the absence of expression of intermediate $(O P N)$ and late $(O C N)$ markers of differentiation, confirmed the early stage of the process.

Osteogenic differentiation was completed in all SSEA$4^{+}$hASC-derived pre-OB co-cultures and monocultures in both OST and MIX media but not in ENDO, as demonstrated by the ALP activity profile, the expression and deposition of $O P N$ and $O C N$ and the amount of calcium. Our results with ENDO medium are in agreement with several studies that showed higher ALP activity in co-cultures of MSCs and ECs than in MSC monocultures. However, in those studies, in the absence of osteo-inductive factors (Dex, AA and $\beta \mathrm{GP}$ ), the completion of osteogenic differentiation, characterized by an extensive matrix mineralization, was not achieved (Hofmann et al., 2008; Villars et al., 2002). Additionally, the concentration of the osteogenic factors is also of relevance (Ma et al., 2011). A 1:1 mixture of ENDO and OST media did not support the osteogenic differentiation of hMSCs in co-culture with ECs and in monoculture, in opposition to the increased mineralization observed in OST medium. These findings reinforce the need for defining a suitable composition of the medium that, besides sustaining the maintenance of the endothelial phenotype, would foster the crosstalk between ECs and osteogenic cells. This was attained with our MIX medium, which contained the required factors at the standard optimum concentrations.

The differences observed for the varied cell ratios also suggest an additional modulatory effect of the SSEA-4 ${ }^{+}$ hASC-derived ECs over SSEA-4 ${ }^{+}$hASC-derived pre-OBs differentiation. A significantly higher ALP activity (day 7), expression of OPN (day 7) and OCN (day 14) and calcium content was detected in the 75:25 cell ratio in relation to SSEA- $4^{+}$hASC-derived pre-OB monocultures (in both MIX and OST media) and the other cell ratios (in MIX medium). The same trend was observed for the levels of VEGF, known to be secreted by osteoblasts in response to the presence of ECs (Street and Lenehan, 2009). Interestingly, significantly higher levels of VEGF were also detected for the 50:50 group in MIX medium in comparison to 25:75 and SSEA- $4^{+}$hASCderived pre-OB monocultures in MIX and OST media. However, no differences were observed regarding the osteogenic markers, suggesting that the minimal number of SSEA-4 ${ }^{+}$ hASC-derived ECs at which cells in co-culture synergistically communicate to support the full differentiation of the pre-OBs and the maintenance of the ECs phenotype, was reached.

Based on our observations and on previous knowledge in deciphering the intimate dialogue between boneforming cells and microvascular-like ECs, we propose a model of the cellular interactions responsible for superior osteogenic maturation that occurs in the presence of a specific SSEA-4 ${ }^{+}$hASC-derived ECs:SSEA-4 ${ }^{+}$hASCderived pre-OBs cell ratio in MIX medium (Figure 6B). We suggest that SSEA-4 ${ }^{+}$hASC-derived ECs signalling synergizes with the conditioned medium to enhance the osteogenic differentiation of SSEA- ${ }^{+}$hASC-derived preOBs during the culture. This leads to increased VEGF release by the pre-OBs/differentiated cells, influencing the SSEA- $4^{+}$hASC-derived ECs phenotype and growth. As a consequence, the higher percentages of SSEA- $4^{+}$hASCderived ECs are likely to be maintained by the VEGF levels present in the conditioned medium, through a VEGFassociated signalling loop. However, our data suggest that VEGF is not the only player in modulating these interactions. Therefore, VEGF is requisite, but not necessarily sufficient, to sustain the activation of the SSEA-4 ${ }^{+}$ hASC-derived ECs modulatory cascade in some media such as OST.

\section{Conclusion}

The finding presented here allow the definition of the ideal combination of SSEA- ${ }^{+}$hASC-derived pre-OBs and ECs, as well the conditions necessary to maximize their potential under the context of engineering vascularized bone tissue. By co-culturing SSEA- $4^{+} \mathrm{hASCs}^{+}$-derived ECs and pre-OBs at an initial rate $>50: 50$ in a mixture of standard endothelial maintenance and osteogenic differentiation media, cells synergistically communicate to support the full differentiation of pre-OBs and the maintenance of the EC phenotype. Therefore, SSEA- $4^{+}$ hASCs can act as an optimal cellular source to build a bone TE construct combining precommitted cells into the osteogenic lineage and fully differentiated ECs with the potential to promote the regeneration of vascularized bone tissue. 


\section{Acknowledgements}

The authors are grateful to the Portuguese Foundation for Science and Technology (FCT) for personal grants SFRH/BD/ 42968/2008 through the MIT-Portugal Programme (SMM). The research leading to these results has received funding from the MIT/ECE/0047/2009 project, the European Union Seventh Framework Programme (Grant No. FP7/2007-2013, under Grant
Agreement No. REGPOT-CT2012-316331-POLARIS) and the European Research Council (Advanced Grant No. ERC-2012AdG_20120216-321266 for the project ComplexiTE).

\section{Conflicts of interest}

The authors declare no conflicts of interest.

\section{References}

Amosi N, Zarzhitsky S, Gilsohn E et al. 2012; Acidic peptide hydrogel scaffolds enhance calcium phosphate mineral turnover into bone tissue. Acta Biomater 8: 2466-2475.

Astori G, Vignati F, Bardelli S et al. 2007; In vitro and multicolor phenotypic characterization of cell subpopulations identified in fresh human adipose tissue stromal vascular fraction and in the derived mesenchymal stem cells. $J$ Transl Med 5: 55 .

Bidarra SJ, Barrias CC, Barbosa MA et al. 2011; Phenotypic and proliferative modulation of human mesenchymal stem cells via crosstalk with endothelial cells. Stem Cell Res 7: 186-197.

Chung MT, Liu C, Hyun JS et al. 2013; CD90 (Thy-1)-positive selection enhances osteogenic capacity of human adipose-derived stromal cells. Tissue Eng A 19: 989-997.

Deckers MM, Karperien M, van der Bent C et al. 2000; Expression of vascular endothelial growth factors and their receptors during osteoblast differentiation. Endocrinology 141: 1667-1674.

Dohle E, Fuchs S, Kolbe M et al. 2010; Sonic hedgehog promotes angiogenesis and osteogenesis in a coculture system consisting of primary osteoblasts and outgrowth endothelial cells. Tissue Eng A 16: 1235-1237.

Duffy GP, Ahsan T, O’Brien T et al. 2009; Bone marrow-derived mesenchymal stem cells promote angiogenic processes in a time- and dose-dependent manner in vitro. Tissue Eng A 15: 2459-2470.

Ferreira LS, Gerecht S, Shieh HF et al. 2007; Vascular progenitor cells isolated from human embryonic stem cells give rise to endothelial and smooth muscle like cells and form vascular networks in vivo. Circ Res 101: 286-294.

Fischer LJ, McIlhenny S. Tulenko T et al. 2009; Endothelial differentiation of adipose-derived stem cells: effects of endothelial cell growth supplement and shear force. J Surg Res 152: 157-166.

Grellier M, Bordenave L, Amédée J 2009; Cell-to-cell communication between osteogenic and endothelial lineages: implications for tissue engineering. Trends Biotechnol 10: 562-571.

Grellier M, Ferreira-Tojais N, Bourget C et al. 2009; Role of vascular endothelial growth factor in the communication between human osteoprogenitors and endothelial cells. J Cell Biochem 106: 390-398.

Groeneveld EH, Burger EH 2000; Bone morphogenetic proteins in human bone regeneration. Eur J Endocrinol 142: 9-21.
Guillotin B, Bareille R, Bourget C et al. 2008; Interaction between human umbilical vein endothelial cells and human osteoprogenitors triggers pleiotropic effect that may support osteoblastic function. Bone 42: 1080-1091.

Hofmann A, Ritz U, Verrier S et al. 2008; The effect of human osteoblasts on proliferation and neo-vessel formation of human umbilical vein endothelial cells in a longterm 3D co-culture on polyurethane scaffolds. Biomaterials 29: 4217-4226.

Huang Z, Ren PG, Ma T et al. 2010; Modulating osteogenesis of mesenchymal stem cells by modifying growth factor availability. Cytokine 51: 305-310.

Hutley LJ, Herington AC, Shurety W et al. 2001; Human adipose tissue endothelial cells promote preadipocyte proliferation. Am J Physiol Endocrinol Metab 281: E1037-1044.

Kaigler D, Krebsbach PH, Wang Z et al. 2006; Transplanted endothelial cells enhance orthotopic bone regeneration. J Dent Res 85: 633-637.

Kaigler D, Krebsbach PH, West ER et al. 2005; Endothelial cell modulation of bone marrow stromal cell osteogenic potential. FASEB $J$ 19: 665-667.

Koob S, Torio-Padron N, Stark GB et al. 2011; Bone formation and neovascularization mediated by mesenchymal stem cells and endothelial cells in critical-sized calvarial defects. Tissue Eng A 17: 311-321.

Levi B, Wan DC, Glotzbach JP et al. 2011; CD105 protein depletion enhances human adipose-derived stromal cell osteogenesis through reduction of transforming growth factor $\beta 1$ (TGF- $\beta 1$ ) signalling. $J$ Biol Chem 286: 39497-39509.

Livak KJ, Schmittgen TD 2001; Analysis of relative gene expression data using realtime quantitative PCR. Methods 25: 402-408.

Ma J, van den Beucken JJJP, Yang F et al. 2011; Coculture of osteoblasts and endothelial cells: optimization of culture medium and cell ratio. Tissue Eng C Methods 17: 349-357.

Marom R, Shur I, Solomon R et al. 2005; Characterization of adhesion and differentiation markers of osteogenic marrow stromal cells. J Cell Physiol 202: 41-48.

Mihaila SM, Frias AM, Pirraco RP et al. 2013; Human adipose tissue-derived SSEA-4 subpopulation multi-differentiation potential towards the endothelial and osteogenic lineages. Tissue Eng A 19: 235-246.
Pfaffl MW 2001; A new mathematical model for relative quantification in real-time RT-PCR. Nucleic Acids Res 29: e45.

Pirraco RP, Marques AP, Reis RL 2010; Cell interactions in bone tissue engineering. $J$ Cell Mol Med 1-2: 93-102.

Porter RM, Huckle WR, Goldstein AS 2003; Effect of dexamethasone withdrawal on osteoblastic differentiation of bone marrow stromal cells. $J$ Cell Biochem 90: 13-22.

Rada T, Reis RL, Gomes ME 2011; Distinct stem cell subpopulations isolated from human adipose tissue exhibit different chondrogenic and osteogenic differentiation potential. Stem Cell Rev Rep 7: 64-76.

Rodan GA 1992; Introduction to bone biology. Bone 13(suppl 1): S3-6.

Rouwkema J, Westerweel PE, de Boer J et al. 2009; The use of endothelial progenitor cells for prevascularized bone tissue engineering. Tissue Eng A 15: 2015-2027.

Santos MI, Unger RE, Sousa RA et al. 2009; Crosstalk between osteoblasts and endothelial cells co-cultured on a polyca prolactone-starch scaffold and the in vitro development of vascularization. Biomaterials 30: 4407-4415.

Street J, Lenehan B 2009; Vascular endothelial growth factor regulates osteoblast survival - evidence for an autocrine feedback mechanism. $J$ Orthop Surg Res 4: 19.

Unger RE, Sartoris A, Peters K et al. 2007; Tissue-like self-assembly in cocultures of endothelial cells and osteoblasts and the formation of microcapillary-like structures on three-dimensional porous biomaterials. Biomaterials 28: 3965-3976.

Valenzuela CD, Allori AC, Reformat DD et al. 2013; Characterization of adipose-derived mesenchymal stem cell combinations for vascularized bone engineering. Tissue Eng A 19: 1373-1385.

Villars F, Guillotin B, Amédée T et al. 2002; Effect of HUVEC on human osteoprogenitor cell differentiation needs heterotypic gap junction communication. Am J Physiol Cell Physiol 282: C775-785.

Xue Y, Xing Z, Hellem S et al. 2009; Endothelial cells influence the osteogenic potential of bone marrow stromal cells. Biomed Eng Online 8: 34.

Zimmerlin L, Donnenberg VS, Rubin JP et al. 2013; Mesenchymal markers on human adipose stem/progenitor cells. Cytometry A 83: 134-140. 


\section{Supporting information on the internet}

The following supporting information may be found in the online version of this article:

Figure S1. Effect of culture conditions on cell morphology. SSEA-4+ hASC-derived ECs maintain their cobblestone-like morphology in ENDO and MIX, whereas in OST they are sparse, not being able to recover during the culture time Figure S2. Relative expression levels of endothelial-specific markers CD31 and $v W F$ for SSEA-4+ hASC-derived EC monocultures in ENDO medium $(\mathrm{A} 1,2)$ and SSEA-4+ hASC-derived pre-OB monocultures in all three culture media $(B 1,2)$ in comparison to EC monocultures at the beginning of the experiment. While SSEA-4+ hASC-derived ECs were characterized by stable expression, SSEA-4+ hASC-derived pre-OBs in ENDO medium lack expression of these markers, as at the beginning of the experiment. Data are expressed as mean $\pm \mathrm{SD} ; \mathrm{n}=3$

Figure S3. Relative expression levels of osteogenic-specific markers OPN and OCN for SSEA-4+ hASC-derived pre-OB monocultures in OST medium (A1, 2) and EC monocultures in all three media (B1, 2) in comparison to SSEA-4+ hASCderived pre-OB monocultures at day 0. While SSEA-4+ hASC-derived pre-OBs gained an osteoblastic-like phenotype, as shown by the significant upregulation of OPN and OCN, SSEA-4+ hASC-derived ECs were characterized by stable gene expression levels. Data are expressed as mean $\pm \mathrm{SD} ; n=3 ; * * * p<0.001$ 